\title{
UTILISATION DES COMPOSANTES DE LA DENSITÉ EN XYLOCHRONOLOGIE
}

\author{
R. KELLER et C. MILLIER \\ Station de Recherches sur la Qualité des Bois, \\ Station de Biométrie, \\ Centre national de Recherches forestières, 54-Nancy \\ Institut national de la Recherche agronomique
}

\section{RESUME}

L'utilisation des composantes de la densité du bois permet d'introduire de nouvelles variables dans la méthode de datation. Cette étude voudrait montrer l'intérêt qu'il y a d'adjoindre à la largeur de cernes des caractéristiques densitométriques obtenues grâce à l'analyse radiographique des bois.

Deux essences ont été considérées: le Sapin pectiné (Abies alba Mill.) et l'Epicéa commun (Picea abies (L.) Karst.) ; les échantillons étudiés ont été prélevés à la tarière de Pressler de $5 \mathrm{~mm}$ dans un peuplement où ces deux essences croissent en mélange. Au total, 100 Sapins et 100 Epicéas ont été sondés.

L'étude xylochronologique montre que, dans le cas du Sapin, la largeur de cernes fournit environ $15 \%$ d'années caractéristiques. Si l'on ajoute à cetie donnée précédente l'écart entre la densité maximale de lannée $n+1$ et la densité minimale de l'année $n$, le pourcentage d'années caractéristiques atteint $37 \%$. Chez l'Epicéa, on en trouve respectivement $7,5 \%$ si l'on considère la largeur de cernes seule, et $26 \%$ si l'on joint à cette donnée la densité maximale annuelle.

On a considéré qu'une année était caractéristique lorsque son pourcentage de coïncidence dépassait $70 \%$. Les observations ont porté sur une période de 27 ans. Dans le cas de l'Epicéa, seules les données densitométriques ont fourni des pourcentages de coïncidence supérieurs à
$80 \%$.

En ouire, puisque l'on disposait de plusieuts vatiables pour chaque année, on a déterminé quelles étaient les liaisons entre ces variables et fait une analyse des composantes principales. On espère qu'une telle méthode de calcul pourrait servir à la datation d'échantillons donnés et à la détermination de la place d'années manquantes par comparaison avec une série xylochronologique de référence.

\section{INTRODUCTION}

Les études de dendrochronologie ont jusqu'à présent été menées à partir des mesures des accroissements annuels des arbres. Les courbes obtenues, souvent après une transformation logarithmique des largeurs de cernes, font ressortir chez les divers arbres les analogies qui existent dans leur comportement vis-à-vis du milieu. 
Les courbes de la variation de la densité du bois en fonction du temps sont susceptibles d'apporter des renseignements nouveaux. Au lieu de n'avoir pour une année qu'une caractéristique, c'est-à-dire la largeur du cerne, on peut déterminer les valeurs d'autres caractéristiques supplémentaires qui sont les mesures de la densité.

Les courbes obtenues par microdensitométrie étant l'image continue de l'évolution de la densité du bois en fonction des années, il est facile de les comparer directement sans qu'il soit besoin au préalable de reporter les mesures sur un graphique. Souvent apparaissent des analogies frappantes dans la forme des courbes. Il peut s'agir d'une seule année ou d'une suite d'années.

Le fait de retrouver, sur de nombreuses courbes enregistrées, des formes semblables aux mêmes années, et à des intervalles identiques, permet de penser que, comme les largeurs de cernes, les caractéristiques de la densité peuvent servir de témoins pour certaines années remarquables. Ceci est d'autant plus intéressant que des années se distinguant par la largeur de leurs cernes ne le font pas forcément aussi pour leur densité, et inversement. La largeur et la densité, dans le cas où elles ne sont pas liées. se complètent et une datation pourra se fonder sur des suites d'années faisant intervenir les deux types de caractéristiques, successivement ou en combinaison, dans les cas où les mesures sur la densité pourront être effectuées.

\section{1. - COMPARAISON DIRECTE A PARTIR DES DONNEES PRISES UNE A UNE}

\section{1. - Matériel et méthode}

Dans notre étude, chaque cerne annuel a été l'objet de plusieurs mesures: sa largeur T. sa densité minimale $d$, sa densité maximale $\mathrm{D}$, et la largeur du cerne $\mathrm{BE}$ dont la densité égale ou dépasse une valeur donnée.

Ces déterminations ont été effectuées à partir de sondages à la tarière de Pressler dans un peuplement mélangé de sapins et d'épicéas reconstitué par plantation après des coupes importantes faites en 1917 qui avaient laissé quelques préexistants. Ces prélèvements n'étaient pas destinés, à l'origine, à servir de matériel pour une étude de xylochronologie, mais seulement pour étudier l'influence de l'éclaircie sur les propriétés du bois.

On se proposait de mettre en évidence des différences dans la qualité du bois formé avant et après léclaircie d'une part, et dans celle du bois formé dans le côté le plus éclairci de l'arbre et dans le côté opposé d'autre part. C'est la raison pour laquelle on a pris, dans chacune des deux parcelles 1 et 2 du peuplement en question, deux échantillons sur chaque arbre. lun $\mathrm{E}$, correspondant au côté le plus éclairci, l'autre $n \mathrm{E}$. lui étant diamétralement opposé (voir figure 1).

Il ne semble pas, ce qui peut paraitre paradoxal et surprenant, qu'il soit possible de tirer des conclusions nettes pour l'instant de l'étude de l'éclaircíe proprement dite qui a eu lieu dans la parcelle 1 en 1959 et dans la parcelle 2 en 1957. Cependant, les calculs faits sur les données mesurées ont montré que l'on pouvait faire mention de résultats annexes au sujet des. quels nous avons pensé qu'il n'y avait pas d'inconvénient à conserver les distinctions prévues dans l'étude de l'éclaircie, c'est-à-dire : parcelle 1 et parcelle 2 , cốté éclairci E et côté non éclairci $n \mathrm{E}$, quitte à faire ensuite des regroupements et des moyennes donnant des résultats plus généraux.

Les carottes obtenues ont un diamètre d'environ $5 \mathrm{~mm}$. Ces carottes sont radiographiées parallèlement au fil du bois; les rayons sont plus ou moins arrêtés par le bois et impressionnent différentiellement le film radiographique sur lequel sont posés les échantillons. Après développement, les zones qui se trouvaient sous du bois dense (bois d'été) apparaissent en taches claires et celles qui étaient sous du bois léger (bois de printemps) en taches sombres. Chaque radiographie porte ainsi l'image fidèle et continue de la densité du bois qu'il reste à transcrire sur des graphiques de la densi.é en fonetion du temps. Cette opération est réa- 
lisée par un microdensitomètre qui traduit fidèlement en courbes continues l'évolution de la densité, en analysant l'opacité du film et en l'équilibrant à chaque instant grâce à un coin optique dont les déplacements sont enregistrés. Chaque radiographie porte en outre un étalon de paliers de densité dont les valeurs servent de repères pour les mesures. La largeur des cernes annuels est facilement mesurable; dans le cas de cernes étroits, on peut choisir un agrandissement convenable.
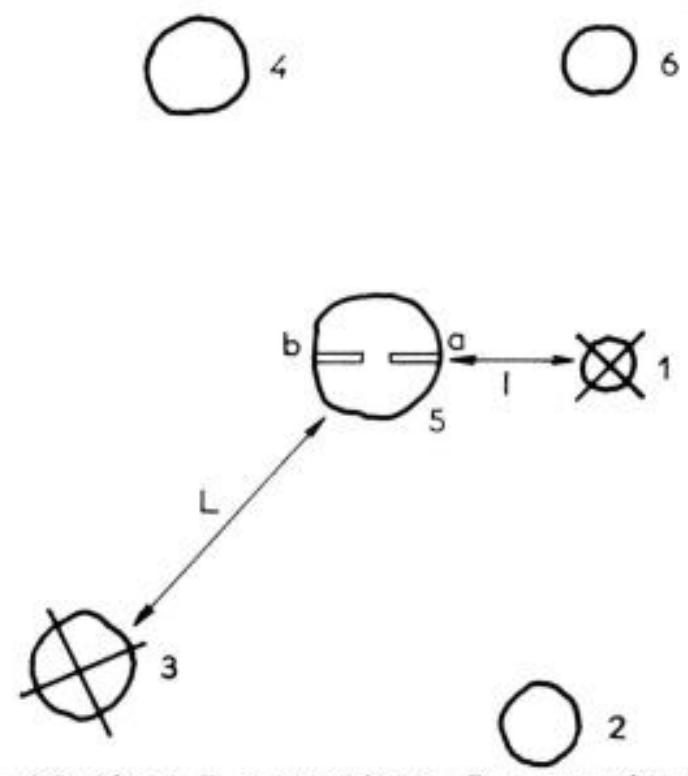

FIG. 1. - Choix des côtés éclaircis $E$, et non éclaircis $n E$, sur un arbre. L'arbre sondé porte le numéro (5). On n'a considéré que les distances relatives I et L pour décider quel était le cồté le plus éclairci sur larbre (5). Ici les arbres (1) et (3) sont partis en éclaircie ; (1) étant plus proche que (3) de (5), c’est à qui a été pris comme côté le plus éclairci, E b, côté nón éclairci nE lui est diamétralement opposé

FıG. 1. - Determination of the thinned side $E$ and of the unthinned side $n E$. The sample tree is the three $\mathrm{n}^{\circ} 5$. The trees $\mathrm{n}^{\circ} \mid$ and 3 have been cut; the distance from 1 to $3:=1 \%$ being longer than the distance from 5 to $3: \leqslant \mathrm{L} \%$, the side $\alpha \mathrm{a} s$ has been considered as $e$ thinned side $s$ and the opposite side \& b* as * unthinned side *

L'idée d'utiliser la densité à des fins xylochronologiques remonte à quelques années (Polge, 1966). Par la suite, les nombreuses courbes de densité n’ont fait que confirmer l'impression que les variations de la densité étaient le résultat assez fidèle des réactions des arbres aux conditions de milieu (se superposant aux caractéristiques propres résultant de l'hérédité de larbre). Par exemple, citons les profils des pins maritimes répartis dans tout le massif landais qui présentent certaines années des formes remarquables, ou encore la mise en évidence de cernes manquants sur des épicéas par la seule considération d'années ou succession d'années dont les profils densitométriques caractéristiques doivent se retrouver à des intervalles déterminés sur la majorité des courbes étudiées (POLGE, KELler, 1969).

La conjugaison de plusieurs aspects caractéristiques chez des arbres d'un même peuple. ment ou de peuplements voisins permet de les * synchroniser \$ avec sûreté. On a pu vérifier sur des coupes microscopiques l'exactitude d'hypothèses de cernes manquants ou partiellement manquants formulées uniquement à cause de l'impossibilité de faire coïncider certaines courbes avec l'ensemble des autres.

Etant donné une grandeur $x$ mesurée sur les graphiques, la comparaison des diverses courbes a été effectuée par l'étude de trois années successives, la valeur de $x$ pour l'année $n$ étant égale, inférieure ou supérieure à celle de l'année $n-1$ ou à celle de l'année $n+1$ pour chacune des caractéristiques. Neuf cas, repérés par les chiffres 1 à 9 , sont possibles. Chaque année de chaque courbe est repérée par l'un des neuf premiers nombres, et il y a autant de nombres que le nombre d'années de l'échantillon étudié diminué de deux. On trouve assez rarement des cas d'égalité, les figures les plus fréquentes étant les 2 , les 7 et surtout les 8 et les 9 (voir tableau 1 ). 


\section{TABLEAU 1}

Tableau des 9 types possibles d'évolution d'une variable mesurée $A$ (largeurs de cernes ou caractéristiques densitométriques). L'ordinateur analyse les suites de trois années et attribue à l'année médiane $n$ le chiffre qui correspond à l'évolution de la grandeur A. Tous les dénombrements ont été ainsi faits de façon automatique

\section{TABLE 1}

Table giving the 9 ways of evolution for a variable $A$ (ringwidth or densitometric characteristics). The computer analysis the 3 years sequences and automatically gives to the median year in the figure corresponding to the way of evolution for the variable A

Chiffre = Figure. $\quad$ Evolution de $\mathrm{A}=$ Evolution of $\mathrm{A}$.

\begin{tabular}{|c|c|c|c|}
\hline Chiffre & $n-1$ & $n \quad n+1$ & Evolution de A \\
\hline 1 & $\longleftarrow$ & $\longrightarrow$ & $A_{n}-1=A_{n}=A_{n}+1$ \\
\hline 2 & & & $A_{n}-1<A_{n}<A_{n}+1$ \\
\hline 3 & 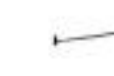 & & $A_{n}-1<A_{n}=A_{n}+1$ \\
\hline 4 & & & $A_{n}-1=A_{n}<A_{n}+1$ \\
\hline 5 & & $\longrightarrow$ & $A_{n}-1=A_{n}>A_{n}+1$ \\
\hline 6 & $\sim$ & $\hookrightarrow$ & $A_{n}-1>A_{n}=A_{n}+1$ \\
\hline 7 & & & $A_{n}-1>A_{n}>A_{n}+1$ \\
\hline 8 & 2 & $\longrightarrow$ & $A_{n}-1>A_{n}<A_{n}+1$ \\
\hline 9 & & $\rightarrow$ & $A_{n}-1<A_{n}>A_{n}+1$ \\
\hline
\end{tabular}

S'il y a $\mathrm{N}$ années, les courbes sont traduites en une suite de $\mathrm{N}-2$ chiffres, Si l'on dispose de $\mathrm{N}$ échantillons, on peut constituer un tableau de $\mathrm{N}$ lignes et $\mathrm{N}-2$ colonnes, chaque colonne correspondant à une année. Il ne reste plus qu’à recenser dans chaque colonne les divers chiffres pour obtenir les proportions des diverses formes de courbes. Ce dénombrement a été fait par un ordinateur à partir des grandeurs mesurées. On a retenu comme caractéristiques les années pour lesquelles au moins $70 \%$ des échantillons présentaient la même évolution dans leurs cousbes individuelles.

L'évolution des variables suivantes a été envisagée ; largeur de cernes $T$, proportion BE/T de largeur de cerne où le bois formé a une densité supérieure à $550 \mathrm{~g} / \mathrm{dm}^{t}$, densité maximale D. densité minimale $d$, moyenne de la densité maximale + densité minimale $\frac{\mathrm{D}+d}{2}$, densité maximale - densité minimale $\mathrm{D}-d$, densité maximale de l'année $n+1$ - densité minimale de l'année $n: \mathrm{D} n+1-d n$, densité maximale de l'année $n-$ densité minimale de l'année $n+1: \mathrm{D} n-d n+1$.

Chaque côté et chaque parcelle ont été étudiés séparément, puis regroupés; de cette façon, les intervalles de confiance des pourcentages observés sont réduits et l'estimation d'un pourcentage sur un nombre plus grand d'échantillons gagne en précision.

Les mesures portent sur 26 années, de 1940 à 1965, les courbes des divers arbres étant synchrones. 


\section{2. - Résultats}

\subsection{1. - Etude des largeurs de cernes $T$ (voir Annexe 1).}

C'est une étude classique. Ici, les comparaisons ont porté sur les données brutes, chaque année étant confrontée à la précédente et à la suivante.

1.211. - Chez le Sapin. - La ressemblance est grande entre les courbes moyennes des deux côtés $a$ et $b$ des arbres d'une parcelle. L'allure générale est décroissante. Les valeurs sont voisines et les variations relatives concordantes au point qu'on pourrait presque superposer les graphiques. Si les deux parcelles se distinguent entre elles par une valeur plus élevée de la largeur des cernes dans la première, elles se ressemblent beaucoup dans leurs variations relatives, les changements brutaux dans les courbes se produisant aux mêmes années. Quatre années se distinguent parmi les autres:

TABLEAU 2

Nombre d'années caractéristiques rapporté au nombré tớal d'années

TABLE 2

Number of characteristic years reported to the total number of years

Pourcentage correspondant $=$ Corresponding percentage.

Type d'évolution (Cf. chiffres tableau 1) = Way of evolution (Cf. figures of table 1).

\begin{tabular}{|c|c|c|c|c|}
\hline & 1943 & 1948 & 1955 & 1956 \\
\hline $\begin{array}{l}\text { Nombre d'années caractéristiques } \\
\text { rapporté au nombre total d'années }\end{array}$ & $139 / 195$ & $171 / 197$ & $169 / 197$ & $144 / 197$ \\
\hline Pourcentage correspondant ...... & 71,3 & 86,8 & 85,7 & 73,1 \\
\hline $\begin{array}{l}\text { Type d'évolution } \ldots \ldots \ldots \ldots \ldots \ldots \\
\text { (Cf. chiffres tableau } 1 \text { ) }\end{array}$ & 9 & 8 & 9 & 8 \\
\hline
\end{tabular}

Deux pourcentages sont élevés (1948 et 1955) et la séquence des années 1954 à 1957 a la forme suivante :

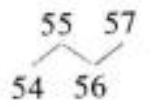

Entre les deux côtés opposés des arbres, la concordance est bonne dans les résultats obtenus :

\section{TABLEAU 3}

Côté éclairci $=$ Thinned side.

Côté non éclairci $=$ Unthinned side.

\begin{tabular}{|c|c|c|c|c|}
\hline & 1943 & 1948 & 1955 & 1956 \\
\hline Còté éclairci $\ldots . . . \ldots \ldots \ldots \ldots \ldots$. & $75 \%$ & $87,6 \%$ & $86,6 \%$ & $74,8 \%$ \\
\hline Côté non éclairci $\ldots \ldots \ldots \ldots \ldots \ldots$ & $67,7 \%$ & $86 \%$ & $85 \%$ & $72 \%$ \\
\hline
\end{tabular}


Entre les deux parcelles, des différences existent, l'une pouvant présenter des années caractéristiques là où l'autre n'en a pas; mais lorsqu'un pourcentage élevé $(>70 \%)$ est observé dans une parcelle, il l'est généralement aussi dans l'autre.

1.212. - Chez l'épicéa. - Il faut noter que la largeur de cerne est moins caractéristique que dans le cas du sapin pour ce qui est des pourcentages de coïncidence, et que, d'autre part, les années caractéristiques peuvent être les mêmes ou différentes (tableau 4).

\section{TABLEAU 4}

\begin{tabular}{|c|c|c|}
\hline & 1955 & 1962 \\
\hline $\begin{array}{c}\text { Nombre d'années caractéristiques rapporté au nombre total } \\
\text { d'années }\end{array}$ & $145 / 200$ & $148 / 200$ \\
\hline Pourcentage correspondant,$\ldots \ldots \ldots \ldots \ldots \ldots \ldots \ldots$, & 72,5 & 74 \\
\hline $\begin{array}{l}\text { Type d'évolution } \ldots \ldots \ldots \ldots \ldots \ldots \ldots \ldots \ldots \ldots \ldots \ldots \ldots \ldots \\
\text { (Cf. chiffres tableau i) }\end{array}$ & 9 & 8 \\
\hline
\end{tabular}

Le maximum de largeur en 1955 se trouve à la fois chez le sapin et lépicéa. 1943, 1948, et 1956 caractérisent le sapin, et 1962, l'épicéa. Les courbes moyennes des largeurs de cernes des épicéas sont très semblables pour les deux côtés opposés des arbres et pour les deux parcelles. Malgré les différences entre les deux essences quant aux années caractéristiques, l'évolution de la largeur de cernes présente des analogies et les courbes moyennes des sapins et des épicéas de la parcelle 2, par exemple, ont un aspect assez net de parenté. On relève donc dans un ordre de ressemblance de moins en moins prononcé les couples suivants : côtés opposés des mêmes arbres, parcelle 1 et parcell 22 pour une même essence, épicéas et sanins dans une même parcelle.

\subsection{2. - Etude de la densité maximale $D$ (voir Annexe 2).}

Le même procédé est appliqué à ces nouvelles données (sans qu'il y ait eu de transformations).

1.221. - Chez le sapin. - D'une façon générale, la densité maximale décroít de 1940 à 1966. Les courbes moyennes des deux côtés opposés des mêmes arbres sont presque superposables. D'une parcelle à l'autre, les analogies sont encore très grandes. Au total, trois années remarquables peuvent être retenues (tableau 5).

TABLEAU 5

\begin{tabular}{|c|c|c|c|}
\hline & 1954 & 1959 & 1964 \\
\hline 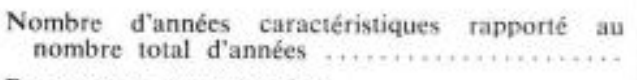 & $143 / 198$ & $161 / 198$ & $148 / 198$ \\
\hline Pourcentage correspondant $\ldots \ldots \ldots \ldots \ldots \ldots \ldots$ & 72,2 & 81,3 & 74,7 \\
\hline $\begin{array}{l}\text { Type d'évolution } \ldots \ldots \ldots \ldots \ldots \ldots \ldots \ldots \ldots \ldots \\
\text { (Cf. chiffres tableau i) }\end{array}$ & 8 & 9 & 9 \\
\hline
\end{tabular}


Ce sont des années différentes de celles que l'on trouve dans le cas des largeurs de cernes; pour la période de 1940 à 1967, on se trouve en présence de sept années caractéristiques si l'on retient à la fois la largeur de cernes et la densité maximale.

1.222. - Chez l'épicéa. - A l'inverse de ce aui se pase chez le sapin, la courbe des densités maximales tend à croitre. Comme dans le cas du sapin, la similitude entre les courbes moyennes des deux côtés opposés des arbres est très grande, et un peu moins nette dans le cas des courbes moyennes darbres des deux parcelles.

Bien que les tendances générales des courbes des deux essences soient divergentes, on retrouve de nombreux points communs dans les variations relatives annuelles qui pourraient traduire une sensibilité analogue aux conditions de milieu, venant se superposer à l'évolution propre de chaque essence.

Cinq années caractéristiques peuvent être relevées (tableau 6).

TABLEAU 6

\begin{tabular}{|c|c|c|c|c|c|}
\hline & 1944 & 1954 & 1959 & 1960 & 1964 \\
\hline 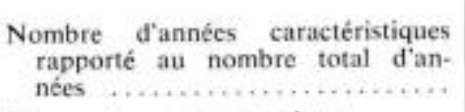 & $134 / 185$ & $169 / 201$ & $172 / 200$ & $148 / 201$ & $154 / 199$ \\
\hline Pourcentage correspondant..... . & 72,5 & 84,2 & 86 & 73,6 & 77,4 \\
\hline $\begin{array}{l}\text { Type d'évolution } \\
\text { (Ć } f \text {. chiffres tableau } 1)\end{array}$ & 8 & 8 & 9 & 8 & 9 \\
\hline
\end{tabular}

On peut remarquer que le nombre en est plus grand que celui qui est relatif aux largeurs de cernes pour cette essence ( 5 contre 2$)$ et que les années caractéristiques pour la densité maximale sont distinctes des années caractéristiques pour les largeurs de cernes; au total, les largeurs de cernes et la densité maximale livrent sept années remarquables pour une période de 27 années.

\subsection{3. - Etude de la densité minimale d (voir Annexe 3).}

Dans cette étude, il semble que la densité minimale fournisse en moyenne des valeurs relatives moins contrastées d'une année à l'autre que la densité maximale.

1.231. - Chez le sapin. - Les courbes ont une tendance générale croissante, nette, mais faible. Les courbes moyennes des côtés opposés des arbres sont très semblables ainsi que les courbes moyennes des arbres des deux parcelles. Les similitudes portent aussi bien sur la forme générale des courbes que sur les diverses valeurs absolues de la densité.

On n'a pas trouvé, pour cette essence, d'année caractéristique commune aux deux côtés opposés des arbres. Il n'y a qu'un côté (le côté non éclairci) qui fournit un pourcentage d'arbres atteignant $70 \%$ et dont la densité minimale est plus forte en 1956, qu'en 1955 et 1957. 
1.232. - Chez lépicéa. - Comme dans le cas du sapin, les courbes moyennes des deux côtés opposés des arbres, ou des deux parcelles, sont très comparables quant à leur forme et aux valeurs absolues des densités. Ceci est encore vrai de l'aspect général des courbes moyennes des deux essences, sapins et épicéas, qui ont même comportement. Mais la densité minimale des sapins est supérieure à celle des épicéas.

Chez l'épicéa non plus, il n'est pas possible de trouver d'année caractéristique générale convenant à la fois pour les deux parcelles et les deux côtés opposés des arbres.

On peut, toutefois, remarquer qu'aussi bien chez l'épicéa que chez le sapin, mais d'une manière plus nette chez le sapin, les densités minimales ont des valeurs élevées en 1945 et 1956.

1.24. - Etude de lécart $D \mathrm{n}$ - dn entre la densité maximale et la densité minimale de l'année n (voir Annexe 4).

Les caractéristiques suivantes font intervenir deux des grandeurs étudiées précédemment pour voir dans quelle mesure les nouvelles combinaisons permettraient d'observer, soit des années caractéristiques nouvelles, soit des pourcentages de coïncidence plus élevés que ceux qui sont fournis par les données simples. La première de ces caractéristiques envisagées est l'écart de densité entre le bois initial et le bois final d'une même année.

1.241. - Chez le sapin. - On pourrait faire les mêmes remarques sur les ressemblances entre les diverses courbes moyennes que dans les cas précédents A, B et C. Avec l'augmentation de son âge, le sapin des deux parcelles voit l'écart $\mathrm{D} n-d n$ diminuer. Au début de la période 1940-1966, D $n-d n$ était voisin de $440 \mathrm{~g} / \mathrm{dm}^{3}$ en parcelle 1 et $460 \mathrm{~g} / \mathrm{dm}^{3}$ en parcelle 2 .

A la fin de la même période, cet écart vaut environ $380 \mathrm{~g} / \mathrm{dm}^{3}$ dans les deux parcelles. Par rapport aux années caractéristiques fournies par la densité maximale Dn seule, l'étude de l'écart $\mathrm{D} n-d n$ semble ici plus avantageuse puisqu'on trouve deux années supplémentaires, 1947 et 1965 (tableau 7).

TABLEAU 7

\begin{tabular}{|c|c|c|c|c|c|}
\hline & 1947 & 1954 & 1959 & 1964 & 1965 \\
\hline $\begin{array}{l}\text { Nombre d'années earactéristiques } \\
\text { rapporté au nombre total d'an- } \\
\text { nées } \ldots \ldots \ldots \ldots \ldots \ldots \ldots \ldots \ldots \ldots \ldots \ldots\end{array}$ & $139 / 198$ & $139 / 198$ & $160 / 196$ & $159 / 198$ & $145 / 197$ \\
\hline Pourcentage correspondant ...... & 70,2 & 70.2 & 81,6 & 80,4 & 73,6 \\
\hline $\begin{array}{l}\text { Type d'évolution } \\
\text { (Cf. chiffres tableat i) }\end{array}$ & 9 & 8 & 9 & 9 & 8 \\
\hline
\end{tabular}

Le pourcentage de 1954 est un peu plus faible que dans le cas de Dn $(70,2 \%$ contre $72,2 \%$ ), celui de 1959 est le même dans les deux cas $(81,3 \%$ et $81,6 \%$ ); en 1964 , le fait de considérer $\mathrm{D} n-d n$ au lieu de $\mathrm{D} n$ seul augmente le pourcentage des coïncidences (il passe de $74,7 \%$ à $80,4 \%$ ). Enfin, les deux nouvelles années font passer, pour cette essence, le nombre des années caractéristiques à 12 sur 27 : $1943(\mathrm{~T}), 1947(\mathrm{D} n-d n), 1948(\mathrm{~T}), 1954$ deux fois (Dn et $\mathrm{D} n-d n), 1955$ (T). 
1956 (T), 1959 deux fois (Dn et $\mathrm{D} n-d n$ ), 1964 deux fois ( $\mathrm{D} n$ et $\mathrm{D} n-d n$ ), 1965 $(\mathrm{D} n-d n)$. Il n'est pas surprenant de trouver des années communes pour les caractères $\mathrm{D} n$ et $\mathrm{D} n-d n$, les deux grandeurs étant évidemment liées, ce qui est important c'est de trouver quelle est la combinaison qui fournit les pourcentages les plus élevés.

1242. - Chez lépicéa. - La tendance générale des courbes moyennes est à une légère augmentation de l'écart des densités qui, au début de la période étudiée, vaut environ $420 \mathrm{~g} / \mathrm{dm}^{3}$ pour atteindre, dans les dernières années, $440 \mathrm{~g} / \mathrm{dm}^{3}$. L'année 1944 n'apparaît plus comme caractéristique, mais 1965 le devient alors qu'on ne la trouvait pas dans le cas de $\mathrm{D} n$ (tableau 8).

TABLEAU 8

\begin{tabular}{|c|c|c|c|c|c|}
\hline & 1954 & 1959 & 1960 & 1964 & 1965 \\
\hline $\begin{array}{l}\text { Nombre d'années caractéristiques } \\
\text { rapporté au nombre total d'an- } \\
\text { nées }\end{array}$ & $168 / 201$ & $169 / 200$ & $141 / 201$ & $171 / 198$ & $154 / 194$ \\
\hline Pourcentage correspondant $\ldots . .$. & 83,6 & 84,5 & 70.2 & 86,4 & 79,4 \\
\hline $\begin{array}{l}\text { Type d'évolution } \ldots \ldots \ldots \ldots \ldots \ldots \ldots \\
\text { (Cf, chiffre tableau i) }\end{array}$ & 8 & 9 & 8 & 9 & 8 \\
\hline
\end{tabular}

Le pourcentage de 1954 est un peu plus faible que dans le cas de Dn $(83,6 \%$ au lieu de $84,2 \%)$, de même pour $1959(84,5 \%$ au lieu de $86 \%)$ et $1960(70,2 \%$ au lieu de $73,6 \%)$. En 1964 , il est plus élevé pour la caractéristique $\mathrm{D} n-d n(86,4 \%$ au lieu de $77,4 \%$ ).

\subsection{5. - Etude de la moyenne $(\mathrm{D} n+d n) / 2$ (voir Annexe 5).}

Cette caractéristique n'est pas la densité moyenne réelle du cerne de l'année $n$ qui serait égale à la surface hachurée $\mathrm{S}^{\prime}$ divisée par la largeur de cerne $\mathrm{T}$, mais une valeur qui ne dépend que de $d n$ et $\mathrm{D} n$ (figure 2).

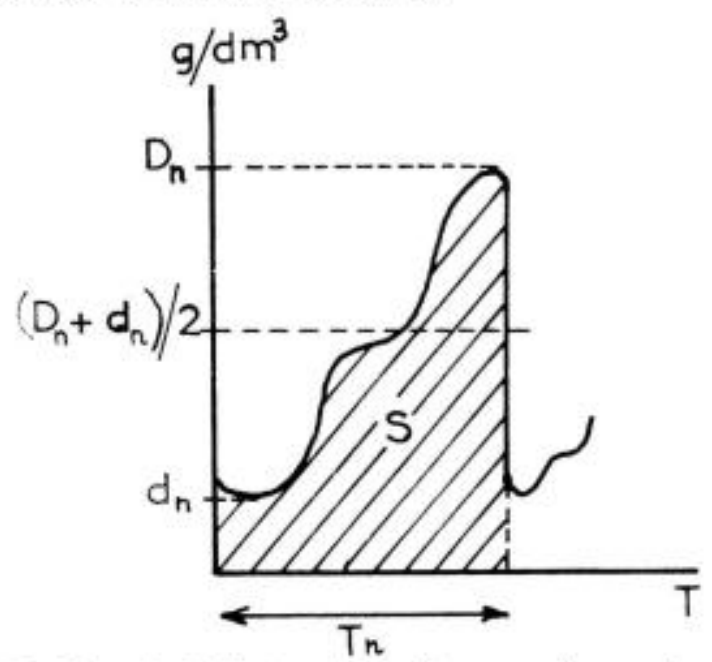

Fig, 2. - Profil densitométrique d'un cerne d'une année $n$

Fic. 2, - Densitometric record of the annual ring 
1.251. - Chez le sapin. - Pour $(\mathrm{D} n+d n) / 2$ aussi on trouve des années déjà citées dans les paragraphes précédents, mais au'si une annéz caractéri tiqu- - ơ 11 : : 1946 pour laquelle $(\mathrm{D} n+d n) / 2$ passe par un minimum. Les pourcentages des années communes sont moins élevés que ceux qui sont relatifs à la densité maxima'e seule Dn ou à l'écart entre les densités maximale et minimale $\mathrm{D} n-d n$ (tableau 9).

TABLEAU 9

\begin{tabular}{|c|c|c|c|}
\hline & 1946 & 1954 & 1959 \\
\hline 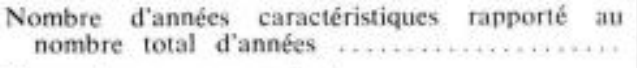 & $149 / 198$ & $143 / 198$ & $152 / 196$ \\
\hline Pourcentage correspondant $\ldots \ldots \ldots \ldots$. & 75,2 & 72,2 & 77,5 \\
\hline $\begin{array}{l}\text { Type d'évolution ................................ } \\
\text { (Cf, chiffres tableau i) }\end{array}$ & 8 & 8 & 9 \\
\hline
\end{tabular}

1.252. - Chez l'épicéa, — Une année supplémentaire apparaît (1949) où (Dn + dn) $/ 2$ atteint un maximum partiel. Toutes les autres années ont déjà été mentionnées (tableau 10).

TABLEAU 10

\begin{tabular}{|c|c|c|c|c|c|}
\hline & 1944 & 1949 & 1954 & 1959 & 1950 \\
\hline $\begin{array}{l}\text { Nombre d'années caractéristiques } \\
\text { rapporté au nombre total d'an- } \\
\text { nées } \ldots \ldots \ldots \ldots \ldots \ldots \ldots \ldots\end{array}$ & $147 / 184$ & $144 / 192$ & $165 / 201$ & $177 / 200$ & $152 / 201$ \\
\hline Pourcentage correspondant $\ldots . .$. & 79,8 & 75 & 82,1 & 88,5 & 80,6 \\
\hline $\begin{array}{l}\text { Type d'évolution } \ldots \ldots \ldots \ldots \ldots \ldots \\
\text { (Cf. chiffre tableau } 1)\end{array}$ & 8 & 9 & 8 & 9 & 8 \\
\hline
\end{tabular}

Les pourcentages observés sont du même ordre de grandeur que ceux de la densité maximale, ou même un peu plus élevés (en 1959: 88.5 \% des échantillons présentent un maximum).

1.26. - Etude du rapport BE/T entre la largeur de cerne BE où le bois formé a une densité égale ou supérieure à $550 \mathrm{~g} / \mathrm{dm}^{3}$ et la largeur totale du cerne $T$ (voir Annexe 6).

Ce rapport fait intervenir à la fois la largeur des cernes annuels et la densité du bois ; elle peut être comparée à la texture traditionnelle.

1.261. - Chez le sapin. - Les courbes relatives au sapin présentent deux périodes où $\mathrm{BE} / \mathrm{T}$ est plus élevé, séparées par les années 1950 à 1955 durant lesquelles ce rapport passe par un minimum. Les valeurs de la parcelle 1 sont moins fortes $(16 \%)$ en moyenne que celles de la parcelle $2(18 \%)$ qui est plus humide; cependant, la forme générale des courbes reste identique. On peut supposer que la plus forte valeur 
de $\mathrm{BE} / \mathrm{T}$ observée dans la parcelle 2 correspond au fait que cette dernière dispose d'un approvisionnement en eau plus abondant que la parcelle 1 , ce qui retarde dans la saison de végétation la formation de trachéides à membranes épaisses et à faibles lumens.

Cette caractéristique n'a pas fourni d'années caractéristiques convenant à la fois pour les deux parcelles: des pourcentages de coïncidence supérieurs à $70 \%$ n'ont pu être observés que dans la parcelle 1. Il est possible qu'en étudiant d'autres niveaux que $550 \mathrm{~g} / \mathrm{dm}^{3}$, on trouve des résultats plus satisfaisants.

1.262. - Chez lépicéa. - Les valeurs de ce rapport sont moins élevées que dans le cas du sapin. La tendance générale de la courbe est à la croissance (parcelle 1: de $8 \%$ à $15 \%$ - parcelle 2 : de $10 \%$ à $15 \%$ ), avec une atténuation de cette tendance dans la période 1950-1955 (pendant laquelle BE/T diminuait chez le sapin). Les valeurs des arbres de la parcelle 2 sont plus fortes que celles des arbres de la parcelle 1, comme dans le cas de l'épicéa. Mais cette caractéristique ne fait pas apparaitre non plus de pourcentages de coïncidence supérieurs à $70 \%$ dans les deux parcelles à la fois.

1.27. - Etude de Técart $\mathrm{D} n-d n+l$ entre la densité maximale $\mathrm{D} n$ d'une année $n$ et la densité minimale $d n+l$ de lannée suivante $n+l$ (voir Annexe 7 ).

Ceci fait intervenir deux années différentes; on a attribué à l'année $n+1$ la valeur de cet écart qui porte sur deux saisons de végétations distinctes (figure 3).

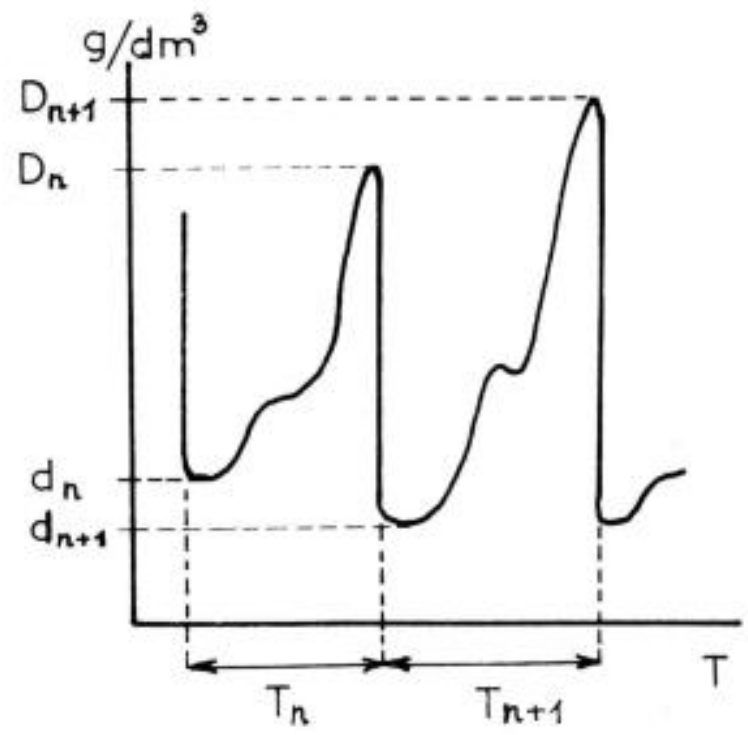

FiG, 3, - Profil densitométrique des cernes de deux années successives $n$ é $n+I$

Fic. 3. - Densitometric record of 2 successive rings $n$ and $n+1$

1.271. - Chez le sapin. - La quantité $\mathrm{D} n-d n+1$ a tendance à décroître, comme du reste $\mathrm{D} n-d n$. Au début de la période, $\mathrm{D} n-d n+1$ vaut environ $430 \mathrm{~g} / \mathrm{dm}^{3}$ dans la parcelle 1 et $450 \mathrm{~g} / \mathrm{dm}^{\text {th }}$ dans la parcelle 2 ; elle décroît dans 
les deux parcelles pour arriver, en moyenne, aux environs de $400 \mathrm{~g} / \mathrm{dm}^{3}$ à la fin des 27 années étudiées.

Les pourcentages de coïncidence dépassent $70 \%$ dans deux cas: en 1948 et en 1960 (tableau 11).

TABLEAU 11

\begin{tabular}{|c|c|c|}
\hline & 1948 & 1960 \\
\hline $\begin{array}{c}\text { Nombre d'années caractéristiques rapporté au nombre total } \\
\text { d'années }\end{array}$ & $149 / 198$ & $151 / 197$ \\
\hline Pourcentage correspondant $\ldots \ldots \ldots \ldots \ldots \ldots \ldots \ldots \ldots \ldots$ & 75,3 & 76.7 \\
\hline $\begin{array}{l}\text { Type d'évolution } \ldots \ldots \ldots \ldots \ldots \ldots \ldots \ldots \ldots \ldots \ldots \ldots \ldots \ldots \ldots \\
\text { (Ćf. chiffres tableau i) }\end{array}$ & 9 & 8 \\
\hline
\end{tabular}

Ces deux années correspondent à 1947 et 1959 trouvées au paragraphe traitant de $\mathrm{D} n-d n$, et pour lesquelles $\mathrm{D} n$ présente un maximum. Dans l'étude de $\mathrm{D} n$ seule. l'année 1947 n'était pas ressortie comme caractéristique, alors que l'association avec $d n$ ou $d n+1$ la fait apparaitre comme telle.

1.272. - Chez. l'épicéa, - Chez cette essence, l'écart $\mathrm{D} n-d n+1$ reste assez constant et oscille autour d'une valeur moyenne voisine de $430 \mathrm{~g} / \mathrm{dm}^{3}$. Sur les quatre années remarquables, l'une a un coefficient particulièrement élevé car l'influence de l'année 1959, pour laquelle $\mathrm{D} n$ a déjà fourni un pourcentage de $86 \%$, se fait sentir, tout en étant renforcée par l'introduction de $d n+1$ (1960 où la densité $d n+1$ passe par un minimum partiel) (tableau 12).

TABLEAU 12

\begin{tabular}{|c|c|c|c|c|}
\hline & 1945 & 1955 & 1960 & 1961 \\
\hline $\begin{array}{l}\text { Nombre d'années caractéristiques } \\
\text { rapporté au nombre total d'an- } \\
\text { nées } \ldots \ldots \ldots \ldots \ldots \ldots \ldots \ldots \ldots\end{array}$ & $149 / 187$ & $157 / 201$ & $179 / 200$ & $151 / 201$ \\
\hline Pourcentage correspondant ...... & 79,6 & 78,2 & 89,5 & 75,2 \\
\hline $\begin{array}{l}\text { Type d'évolution } \ldots . . . \ldots \ldots \ldots \ldots . . . . . . . \\
\text { (Cf. chiffres tableau 1) }\end{array}$ & 8 & 8 & 9 & 8 \\
\hline
\end{tabular}

1.28. - Etude de l'écart $\mathrm{D} n+I-d n$ entre la densité maximale $\mathrm{D} n+l$ d'une année et la densité minimale dn de lannée précédente $n$ (voir Annexe 8).

Cette quantité fait intervenir deux années différentes, comme la précédente (voir figure 3); en fait, ici les caractéristiques composantes $\mathrm{D} n+1$ et $d n$ sont des densités de bois formé à deux périodes séparées par deux saisons de végétation, le bois de densité minimale apparaissant tout au début du départ de la végétation, alors que le bois de densité maximale est élaboré juste avant l'arrêt de la végétation. 
1.281. - Chez le sapin. - L'écart, d’une façon générale, diminue et passe de $440 \mathrm{~g} / \mathrm{dm}^{\text {: }}$ à $410 \mathrm{~g} / \mathrm{dm}^{3}$. Il faut noter parmi les six années caractéristiques, l'année 1958 , intermédiaire entre 1957 et 1959 ; c'est le seul cas où une année se distingue parce qu'elle est intermédiaire entre deux autres, et non pas parce que la quantité étudiée y présente un maximum ou un minimum (tableau 13).

On obtient le double d'années caractéristiques (six au lieu de trois) par rapport à ce que l'on trouve par la prise en considération de la densité maximale seule, et alors que la densité minimale par elle-même ne fournit aucune année remarquable.

TABLEAU 13

\begin{tabular}{|c|c|c|c|c|c|c|}
\hline & 1946 & 1954 & 1957 & 1958 & 1959 & 1964 \\
\hline $\begin{array}{l}\text { Nombre d'années carac- } \\
\text { téristiques rapporté au } \\
\text { nombre total d'années }\end{array}$ & $145 / 198$ & $155 / 198$ & $147 / 197$ & $140 / 196$ & $151 / 196$ & $148 / 198$ \\
\hline Pourcentagz d'évolution. & 73,3 & 78,3 & 74,6 & 71,4 & 77 & 74,8 \\
\hline $\begin{array}{l}\text { Type d'évolution ....... } \\
\text { (Cf. chiffres tableau i) }\end{array}$ & 8 & 8 & 8 & 2 & 9 & 9 \\
\hline
\end{tabular}

1.282. - Chez lépicéa. - Par rapport à la densité maximale seule, on ne trouve pas d'années supplémentaires. Chez cette essence, la tendance est à une très légère augmentation dans la parcelle 1 et à la stabilité dans la parcelle 2 , l'écart moyen valant environ $430 \mathrm{~g} / \mathrm{dm}^{\prime:}$.

Le pourcentage de 1959 est élevé et dépasse celui qui est relatif à la densité maximale $(89 \%$ contre $86 \%)$; à part 1954, les autres années sont plus discriminantes que dans le cas de la densité maximale seule (tableau 14).

TABLEAU 14

\begin{tabular}{|c|c|c|c|c|c|}
\hline & 1944 & 1954 & 1959 & 1960 & 1964 \\
\hline $\begin{array}{l}\text { Nombre d'années caractéristiques } \\
\text { rapporté au nombre lotal d'an- } \\
\text { nées } \ldots \ldots \ldots \ldots \ldots \ldots \ldots \ldots \ldots \ldots \ldots \ldots \ldots \ldots\end{array}$ & $142 / 183$ & $166 / 201$ & $178 / 200$ & $162 / 201$ & $157 / 199$ \\
\hline Pourcentage correspondant ...... & 77,6 & 82,6 & 89 & 80,6 & 38,9 \\
\hline $\begin{array}{l}\text { Type d'évolution ................. } \\
\text { (Cf. chiffres tableau i) }\end{array}$ & 8 & 8 & 9 & 8 & 9 \\
\hline
\end{tabular}

Il semble que des combinaisons de caractères divers peuvent conduire à des années caractéristiques plus nombreuses et à des pourcentages de coïncidence encore plus élevés que ceux qui sont calculés sur les données simples isolées comme la largeur de cernes, les diverses densités ou les niveaux de densités. Il reste à trouver la combinaison la plus favorable à la découverte de ces années. 


\section{3. - Récapitulation des résultats}

1.31. - Cas du sapin.

La largeur de cernes seule fournit quatre années sur 27, soit un peu moins de $15 \%$ d'années caractéristiques. La largeur de cernes et $\mathrm{D} n+1-d n$ donnent dix années sur 27 , soit à peu près $37 \%$ d'années caractéristiques où le pourcentage de coïncidence dépasse $70 \%$ (tableau 15).

TABLEAU 15

Tableau récapitulatif des pourcentages d'années caractéristiques chez le sapin

TABLE 15

Comprehensive table of the percentage of characteristic years for Norway Spruce

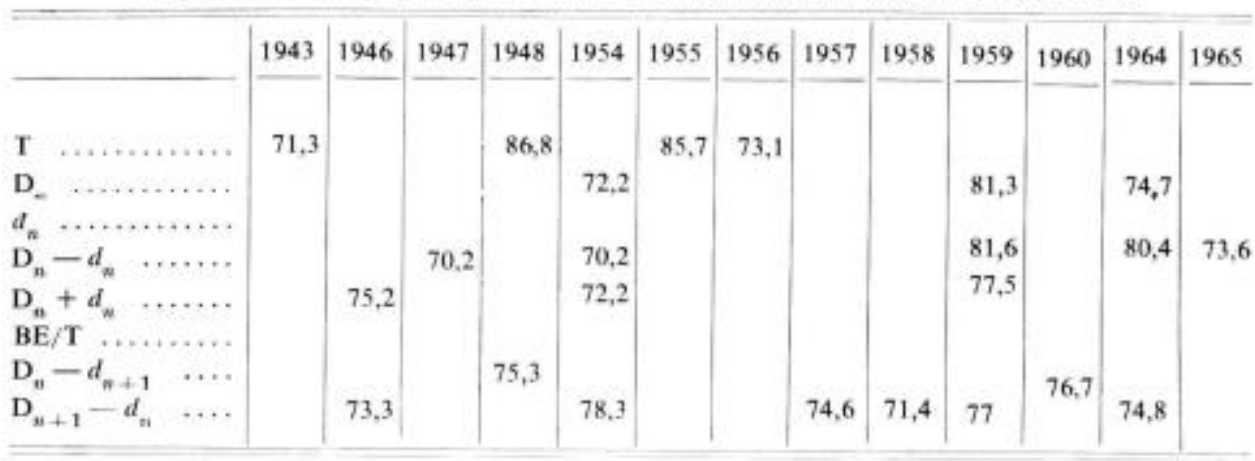

\subsection{2. - Cas de lépicéa.}

La largeur de cernes fournit deux années sur 27, soit un peu moins de $7,5 \%$ d'années caractéristiques. Si l'on adjoint à la largeur de cernes, soit $\mathrm{D} n$, soit $\mathrm{D} n-d n$, doit $\mathrm{D} n+d n$, soit $\mathrm{D} n+1-d n$, on trouve sept années sur 27 , soit presque $26 \%$ d'années caractéristiques où le pourcentage de coïncidence dépasse $70 \%$ (tableau 16).

TABLEAU 16

Tableau récapinulatif des pourcentages af années caractéristiques chez répicéa

TABLE 16

Comprehensive table of the percentage of characteristic years for Silver Fir

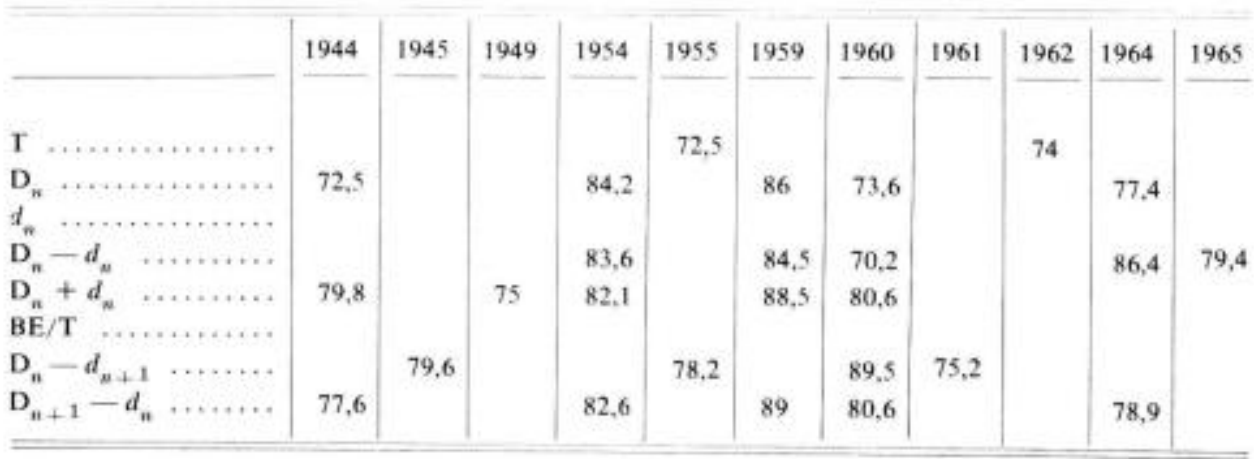

Dans le cas de cette essence, seules les densités fournissent des valeurs supérieures à $80 \%$. 


\subsection{3. - Conclusion.}

Les années remarquables chez les deux essences, sapin et épicéa, sont différentes dans le cas de la largeur de cernes et dans le cas de la densité maximale $\mathrm{D} n$ ou des combinaisons $\mathrm{D} n-d n$ et $\mathrm{D} n+d n$. 11 est donc intéressant d'introduire les caractéristiques densitométriques dans des études de datation des bois à la condition toutefois, qui est presque intuitive, que les diverses grandeurs soient indépendantes les unes des autres.

\section{2. - MISE EN EVIDENCE DE LIAISONS ENTRE LES DIVERSES CARACTERISTIQUES. CALCULS DE CORRELATION}

\section{1. - Pour une parcellé, une essence et un côté donné (voir Annexe 9)}

On peut chercher les liens qui existent entre les diverses caractéristiques mesurées et quelles sont leurs évolutions relatives au cours des années étudiées. Dans ce paragraphe, il s'agit de calculs de corrélation entre les largeurs de cernes $T$, les largeurs de bois d'été $\mathrm{BE}$, les rapports $\mathrm{BE} / \mathrm{T}$, les densités minimales $d$, les densités maximales D, et diverses combinaisons de ces deux dernières, soit, au sein d'une même année, soit à partir de deux années différentes: $(\mathrm{D}+d) / 2, \mathrm{D}-d, \mathrm{D} n-$ $d n+1, \mathrm{D} n+1-d n$, l'indice $n$ repérant l'année. Ces mesures sont les moyennes par année des mesures des arbres compris dans la catégorie envisagée. On dispose donc, pour chacune des neuf caractéristiques, de 26 valeurs correspondant aux années qui vont de 1940 à 1965 (voir Annexe 9).

Nous ne traiterons pas les huit cas possibles (deux parcelles, deux essences, deux côtés), mais nous prendrons deux exemples qui reflètent aussi l'aspect des autres tableaux de corrélation.

\subsection{1. - Chez répicéa, pour le côté éclairci et la parcelle I.}

Chez l'épicéa, la largeur de cerne est liée à la densité minimale, le coefficient de corrélation étant négatif $(r=-0.55)$. Lorsque l'accroissement annuel est grand. la densité minimale du bois de printemps est faible et inversement.

La densité maximale atteinte en fin de végétation semble obéir à une tendance ana'ogue, mais seulement dans la parcelle $1(r=-0,44)$, alors qu'aucune liaison ne peut être mise en évidınce dans le cas des épicéas de la parcelle 2 qui, bien que très proche de la parcelle 1, semble être plus favorisée qu'elle dans son approvisionnement en eau. Le phénomène est le même pour la valeur moyenne $(\mathrm{D}+d) / 2$. Les résultats obtenus à partir des deux prélèvements opposés faits sur chaque arbre sont tout à fait analogues, ce qui se traduit asssi par une corrélation très forte existant, pour une même caractéristique, entre deux côtés opposés d'un même arbre.

La partie BE du cerne où la densité du bois formé dépasse $550 \mathrm{~g} / \mathrm{dm}^{3}$ est fortement liée à la densité maximale $\mathrm{D}(r=0.72)$ dans la parcelle 1 , et moins nettement dans la parcelle 2. Lorsque l'importance du bois d'été est grande, il y a une bonne probabilité pour que la den ité maximale soit forte.

Le rapport $\mathrm{BE} / \mathrm{T}$ est lié nettement à la grandeur $d n$, leur coefficient de corrélation étant positif $(r=0,59)$, un départ de végétation à densité élevée semblant être 
le signe que l'importance du bois d'été par rapport à la largeur totale du cerne sera grande.

\subsection{2. - Chez le sapin, pour le côté éclairci et la parcelle l.}

La largeur de bois d'été BE, dont la limite inférieure est $550 \mathrm{~g} / \mathrm{dm}^{3}$ comme dans le cas de l'épicéa, est liée à la largeur totale de cerne $T$ d'une façon positive $(r=0,86)$. Le résultat est différent de celui qu'on observe chez l'épicéa, pour lequel la liaison entre la largeur de cerne et l'importance du bois d'été a plutôt tendance à être négative dans la parcelle 1 .

Le sapin présente aussi une liaison négative entre la largeur de cerne et la densité minimale ( $r=-0,54$ ), mais, au contraire, de ce qu'on observe chez l'épicéa, la largeur de cerne et la densité maximale atteinte en fin de la saison de végétation varient dans le même sens. En d'autres termes, à une année caractérisée par un accroissement important sur le rayon correspond une densité du bois final élevée. En outre, la liaison positive de l'écart qui existe entre la densité maximale et la densité minimale d'une même année avec la largeur du cerne de l'année en question est bien marquée $(r=0,58)$, ce qui, en quelque sorte, est la synthèse des deux résultats précédents, l'écart $\mathrm{D} n-d n$ augmentant par deux modes différents quand $\mathrm{T}$ augmente. puisque $\mathrm{D} n$ s'accroît et $d n$ diminue.

Comme dans le cas de l'épicéa, la largeur BE de bois possédant une densité supérieure à $550 \mathrm{~g} / \mathrm{dm}^{3}$ est en corrélation positive avec la densité maximale atteinte en fin de saison de végétation $(r=0,68)$. Si l'on considère le rapport $\mathrm{BE} / \mathrm{T}$, on voit qu'il est en liaison avec la densité minimale $d n$ du bois formé au départ de la végétation. Lorsque $d n$ est élevée, le rapport BE/T l'est également, sans que pour autant $\mathrm{D} n$ atteigne une valeur particulièrement forte. La valeur de la densité minimale $d n$ donne ainsi une indication sur ce que sera la proportion de bois à forte densité, tout comme dans le cas de l'épicéa.

\section{2. - Pour une essence, les deux parcelles étant regroupées}

\subsection{1. - Chez lépicéa, pour la moyenne des valeurs des prélèvements opposés (côté} non éclairci et côté éclairci).

Les résultats ne diffèrent pas beaucoup de ce que l'on a déjà observé, mais ils présentent un caractère un peu plus général. II n'est pas vraiment utile de faire une distinction entre les côtés, les tableaux de corrélation relatifs à chaque côté étant analogues à peu de choses près (voir Annexe 10).

La largeur de cerne $\mathrm{T}$ est en corrélation négative avec le rapport BE/T $(r=$ $-0,79)$, la densité minimale annuelle $d n(r=-0,62)$ et la moyenne entre cette dernière quantité et la densité maximale annuelle $(\mathrm{D} n+d n) / 2(r=-0,41)$.

La largeur de bois d'été BE dont la densité dépasse $550 \mathrm{~g} / \mathrm{dm}^{3}$ est liée, comme on peut s'y attendre, à la valeur de la densité maximale $\mathrm{D} n(r=0,63)$, ainsi qu'aux combinaisons $(\mathrm{D} n+d n) / 2(r=0,64)$, $\mathrm{D} n-d n(r=0,57)$. Le rapport $\mathrm{BE} / \mathrm{T}$ est en corrélation positive avec la densité minimale annuelle $d n(r=0,70)$ et la densité maximale annuelle Dn $(r=0,52)$. 
2.22. - Chez le sapin, pour la moyenne des valeurs des prélèvements opposés (côté non éclairci et côté éclairci).

La largeur de cerne $\mathrm{T}$ varie dans le même sens que la largeur de bois d'été $\mathrm{BE}$ $(r=0,83)$, la densité maximale $\mathrm{D} n(r=0,56)$ et l'écart de densité $\mathrm{D} n-d n(r=0,71)$. Ceci est différent de ce qui se passe pour l'épicéa où $\mathrm{T}$ n'est pas liée à $\mathrm{D} n$ (on a vu même que dans la parcelle 1. T et $\mathrm{D} n$ sont en corrélation négative). T est en corrélation négative avec la densité minimale annuelle $d n(r=-0,70)$ comme dans le cas de l'épicéa, mais contrairement à ce qui est observé chez cette dernière essence, $\mathbf{T}$ et $(\mathrm{D} n+d n) / 2$ sont indépendantes.

$\mathrm{BE} / \mathrm{T}$ est liée à la densité minimaie $d n(r=0,60)$ et à $\mathrm{D} n(r=0,43)$.

\subsection{3. - Conclusion partielle.}

Pour résumer ces résultats, on peut dire qu'il semble que, chez l'épicéa, lorsque la largeur de cerne augmente, la proportion de bois à forte densité diminue ; c'est done ce qui pourrait se produire au cours d'une réaction éventuelle à l'éclaircie.

Dans le cas du sapin, on assiste, lorsque la largeur de cerne augmente, à la fois à une diminution de la densité minimale et à une augmentation de la densité maximale, c'est-à-dire à une augmentation des écarts de densité, sans que pour autant la proportion de bois à forte densité s'accroisse.

La réaction à l'éclaircie des deux essences pourrait donc n'être pas tout à fait semblable lorsque $\mathrm{T}$ augmente : la densité diminue en bloc chez l'épicéa, alors que les écarts entre les densités maximale et minimale annuelles augmentent chez le sapin dont la proportion $\mathrm{BE} / \mathrm{T}$ de bois à forte densité diminue puisque sa densité minimale $d n$ et $\mathrm{BE} / \mathrm{T}$ sont en corrélation positive, alors que $d n$ et $\mathrm{T}$ sont en corrélation négative.

Chez les deux essences, le fait de commencer une saison de végétation par une densité minimaie forte laisse présager un rapport $\mathrm{BE} / \mathrm{T}$ élevé.

\section{3. - Corrélations entre les caractéristiques homologues mesurées sur les deux essences}

On peut imaginer de voir quels sont les liens qui existent entre les deux essences croissant en mélange. C'est pourquoi on a fait les calculs des coefficients de corrélation entre les caractéristiques des épicéas et celles des sapins pour les 26 années envisagées. On retiendra en particulier les coefficients qui sont relatifs aux grandeurs homologues, par exemple celui qui traduit la liaison entre la largeur de cerne de l'épicéa et celle du sapin, la densité maximale de l'épicéa et celle du sapin, etc...

Mise à part la quantité $\mathrm{BE}$ qui ne donne pas de résultat significatif, les huit autres grandeurs sont liées entre elles (tableau 17).

Les largeurs de cerne de l'épicéa et celles du sapin font apparaître un coefficient de corrélation $r=0,88$ qui est très significatif ainsi que les densités minimales pour lesquelles $r$ vaut 0,87 . Les densités maximales et diverses de leurs combinaisons avec les densités minimales de la même année ou de l'année précédente ou suivante fournissent des coefficients compris entre 0,56 et 0,64 . 
TABLEAU 17

Coefficient de corrélation entre les grandeurs homologues chez le sapin et lépicéa

TABLE 17

Correlation coefficients between corresponding values for Silver fir and Norway spruce

\begin{tabular}{|c|c|c|c|c|c|c|c|c|c|}
\hline & $\mathrm{T}$ & BE & $\mathrm{BE} / \mathrm{T}$ & $d$ & D & $(\mathrm{D}+d) / 2$ & $D-d$ & D_ $-d_{n+1}$ & $\mathrm{D}_{n+1}-d_{n}$ \\
\hline$r=$ & 0,88 & 0,11 & 0,49 & 0,87 & 0,60 & 0,68 & 0,56 & 0,56 & 0,64 \\
\hline
\end{tabular}

Les deux essences ont des réactions nettement comparables aux actions des facteurs de l'environnement pour ce qui est de la largeur de cerne de la densité minimale et de la densité maximale. La densité minimale se révèle être un facteur sensible, mais lié par ailleurs à la largeur de cernes. C'est peut-être la raison pour laquelle, lors de la recherche des années caractéristiques, et tout au moins dans le cas de l'épicéa, c'est la prise en considération de la densité maximale $\mathrm{D} n$ qui donne le plus d'années caractéristiques différentes de celles qui ont été trouvées grâce à l'étude des largeurs de cernes. En effet, chez cette dernière essence, $\mathrm{T}$ et $\mathrm{D}$ ne sont pas liées. La combinaison à $\mathrm{D} n$ de la densité minimale $d n$, liée à $T$ chez les deux essences, fait apparaître quelques années caractéristiques nouvelles, mais augmente souvent le pourcentage d'années observées déjà mises en évidence grâce à la densité maximale Dn seule.

\section{3. - ESSAI DE DETERMINATION DUUNE METHODE SYNTHETIQUE DE DIFFE- RENCIATION DES ANNEES GRACE A L'ENSEMBLE DES CARACTERISTIQUES MESUREES. ANALYSE DES COMPOSANTES PRINCIPALES}

\section{1. - Méthode}

Chaque année est caractérisée par un certain nombre de grandeurs mesurées dont le nombre pourrait théoriquement être aussi élevé qu'on le désirerait. Elle peut donc être repérée dans un espace dont la dimension est égale au nombre des caractéristiques distinctes mesurées. Or il se trouve que, dans les mesures faites sur les sapins et les épicéas, il en est qui ne sont pas tout à fait distinctes ou indépendantes les unes des autres, soit à cause de leur choix, par exemple $\mathrm{BE}$ et $\mathrm{BE} / \mathrm{T}$, ou $\mathrm{D}, d$ et $\mathrm{D}-d$, soit parce que certaines d'entre elles sont liées sans que l'on puisse s'en douter a priori.

Le but de cette étude est de diminuer le nombre des variables initialement retenues pour ne conserver que celles qui expliquent la plus grande partie de la variation entre les années. On cherche en même temps à rendre maximale cette variation par certaines transformations mathématiques afin de différencier au mieux les années.

Pour cela, les valeurs propres et les vecteurs propres des matrices des coefficients de corrélation totale entre les caractères ont été déterminés, chaque vecteur propre ayant une composante sur les variables initiales. On se limite à un nombre de vecteurs propres suffisant pour expliquer la majeure partic de la variation entre les années, ce que l'on contrôle en déterminant le rapport de la valeur propre calculée, égale à la somme des valeurs propres retenues (en se limitant à $x$ vecteurs propres) à la valeur 
propre théorique totale qui est égale à la somme des termes de la diagonale principale de la matrice des coefficients de corrélation. Les termes de cette diagonale étant tous égaux à 1 , la valeur propre théorique totale vaut $\mathrm{N}$ si $\mathrm{N}$ est le nombre des variables étudiées (Tomassone, 1965).

De cette façon, les années peuvent être séparées les unes des autres, ou au contraire groupées, d'après les valeurs de quelques composantes principales en nombre réduit, elles-mêmes combinaisons linéaires de tous les caractères initiaux mesurés.

La représentation graphique de la position des années en fonction des composantes principales prises deux à deux est possible et permet de mettre en évidence des analogies entre années éloignées, ainsi que des différences entre années proches ou même successives.

\subsection{1. - Cas de l'épicéa.}

L'analyse des composantes principales fait ressortir la parenté existant entre certaines variables initiales ou, à l'inverse, l'indépendance des variables entre elles. En effet, les calculs de corrélation entre les variables initiales $\mathrm{X}$ et les composantes principales $\mathrm{Y}$ retenues montrent une certaine spécialisation de chacune des composantes $\mathrm{Y}$ dans l'explication des variables initiales $\mathrm{X}$, comme par exemple dans le cas suivant où on n’a reporté que les coefficients de corrélation supérieurs à 0,80 (tableau 18).

TABLEAU 18

Coefficients de corrélation entre les données initiales $X_{1}, \ldots, X_{1}$ et les variables $Y_{1}, \ldots, Y_{4}$.

TABLE 18

Correlation coefficients between initial data $X_{1}, \ldots, X_{18}$ and symthetic variables $Y_{1}, \ldots, Y_{4}$.

Epicéa $=$ Norway Spruce.

Moyennes des parcelles 1 et $2=$ Mean values of plots 1 and 2 .

Côté éclairci $=$ Thinned side.

Cóté non éclairci $=$ Unthinned side.

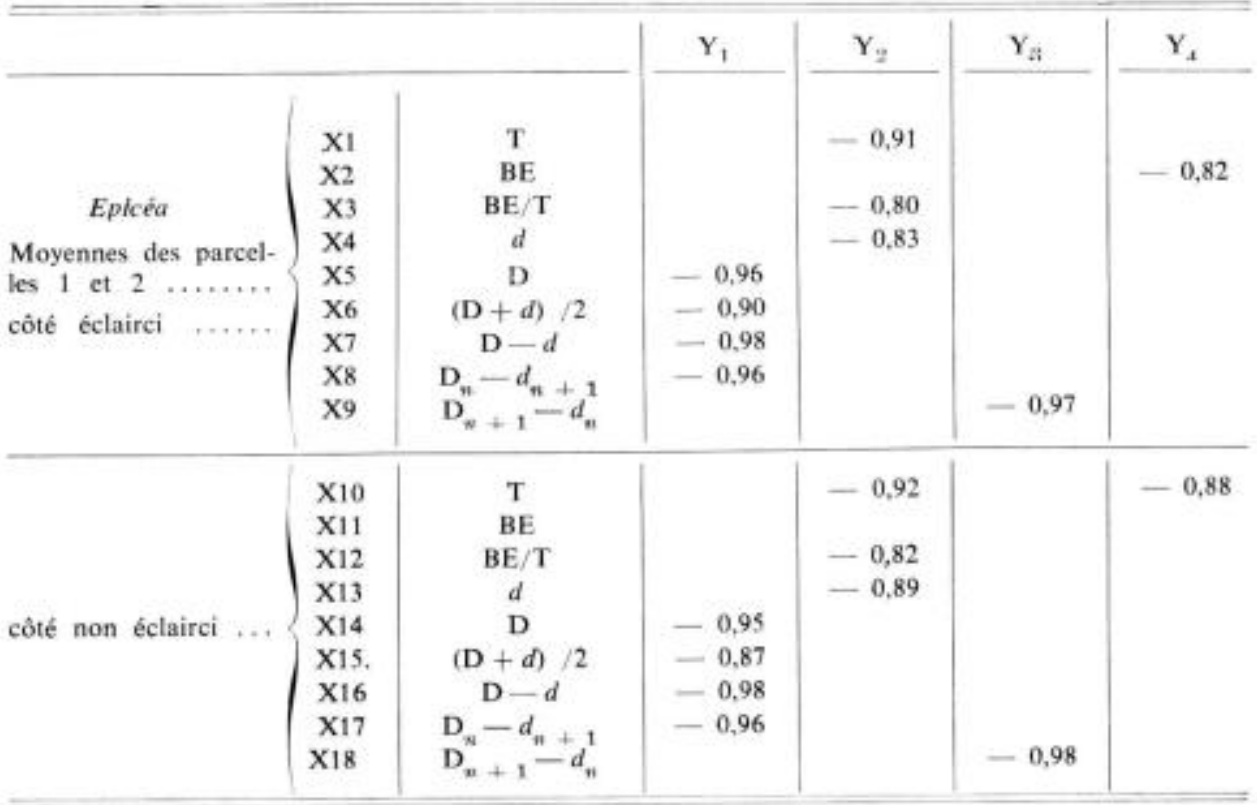


Il s'agit du tableau des corrélations entre 18 variables initiales, 9 pour le côté le plus éclairci de l'arbre, 9 pour le côté opposé, et 4 composantes principales.

La similitude des valeurs pour les deux côtés opposés est frappante, ce qui semblerait du reste montrer que l'éclairement, différent sur les deux côtés des troncs après l'éclaircie, n’a pas joué de rôle sur l'évolution des caractéristiques initia:es mesurées, et autorisé à regrouper ces deux côtés.

En outre, il semble que certaines des variables initiales comme X5, X6, X7, X8 fassent double emploi, la combinaison de $d n$ ou $d n+1$ à $\mathrm{D} n$ n'apportant que peu à l'effet de $\mathrm{D} n$ pris isolément. Ceci se voit aussi sur le graphique des coefficients de corrélation où tout ce qui fait intervenir $\mathrm{D} n$ forme un bloc à part. II suffirait, dans le cas de la composante $\mathrm{Y} 1$, de s'en tenir à la mesure de $\mathrm{D}-d$ par exemple, sans faire intervenir d'autres données relatives à la densité maximaie.

La composante principale Y2 est liée positivement à la largeur de cerne, et négativement à la densité minimale. On retrouve l'antagonisme entre ces deux variables initiales qui était déjà mis en évidence par le calcul des corrélations où l'on avait trouvé un coefficient de $-0,62$ pour l'ensemble des épicéas. Dans l'analyse des composantes principales, retenir comme variable explicative la largeur de cernes ou la densité minimale revient donc à peu près au même pour la différenciation des années. En outre, il n'est pas anormal que, si $\mathrm{T}$ est liée positivement à $\mathrm{Y} 2, \mathrm{BE} / \mathrm{T}$ le soit négativement. Cette dernière variable n'apporte rien à l'analyse.

La variable initiale $\mathrm{D} n+1-d n$, qui représente l'écart de densité entre le bois formé à la fin de la saison de végétation de l'année $n+1$ et celui du début de la saison de végétation de l'année précédente $n$, est fortement liée à une composante principale spéciale Y3. On se rappellera que, si chez l'épicéa, cette grandeur n'a pas fait apparaître d'années caractéristiques nouvelles, elle a aug̣menté le pourcentage d'années caractéristiques déjà trouvées à l'aide de la densité maximale $\mathrm{D} n$ seule. On pourra remarquer que cette grandeur $\mathrm{D} n+1-d n$ n'est pas liée, chez cette essence, aux autres variables initiales.

Enfin, une nouvelle composante principale, Y4, apparaît pour rendre compte de l'action de la variable mesurant la largeur BE du bois d'été dont la densité dépasse $550 \mathrm{~g} / \mathrm{dm} 3$. Cette donnée n'avait pas été utilisée sous cette forme pour déterminer les années caractéristiques; comme chez l'épicéa, elle n'est pas liée à la valcur de la largeur de cernes, il aurait peut-être été utile de la prendre en considération dans leur recherche.

La composante principale $Y_{1}$ permet d'expliquer $54 \%$ de la variation entre les années, les composantes suivantes $\mathrm{Y} 2, \mathrm{Y} 3, \mathrm{Y} 4$ ajoutent respectivement $22 \%, 12 \%$, $7 \%$, si bien qu'au total, les quatre composantes expliquent $95 \%$ de la variation entre les 26 années. L'analyse précédente montre qu’au lieu d'avoir mesuré neuf caractères, on aurait pu se contenter de n'en prendre que quatre qui seraient l'écart de densité $\mathrm{D} n-d n$, la largeur de cernes $\mathrm{T}$ (ou la densité minimale $d n$ ), l'écart de densité entre deux saisons de végétation $\mathrm{D} n+1-d n$ et la largeur de bois d'été $\mathrm{BE}$.

\subsection{2. - Cas du sapin.}

Dans le cas du sapin, si l'on conserve tout d'abord, comme chez I'épicéa, la distinction entre côté éclairci et côté non éclairci, on obtient le tableau des corrélations 
entre les 18 variables initiales et les trois composantes principales qui suffisent à expliquer $93 \%$ de la variation entre les années (tableau 19).

TABLEAU 19

Coefficients de correlation entre les données initiales $X_{1} \ldots, X_{1}$ s et les variables synthétiques $Y_{1}, \ldots$, $Y_{: \mathrm{s}}$

TABLE 19

Correlation coefficients between inirial data $X_{1}, \ldots, X_{1 \times}$ and synthotic variables $Y_{1}, \ldots, Y_{3}$.

Sipin $=$ Silver Fir.

Moyennes des parcelies 1 et 2 = Mean valuss of plots 1 and 2 .

Côté éclairci $=$ Thinned side.

Côté non éclairci $=$ Unthinned side.

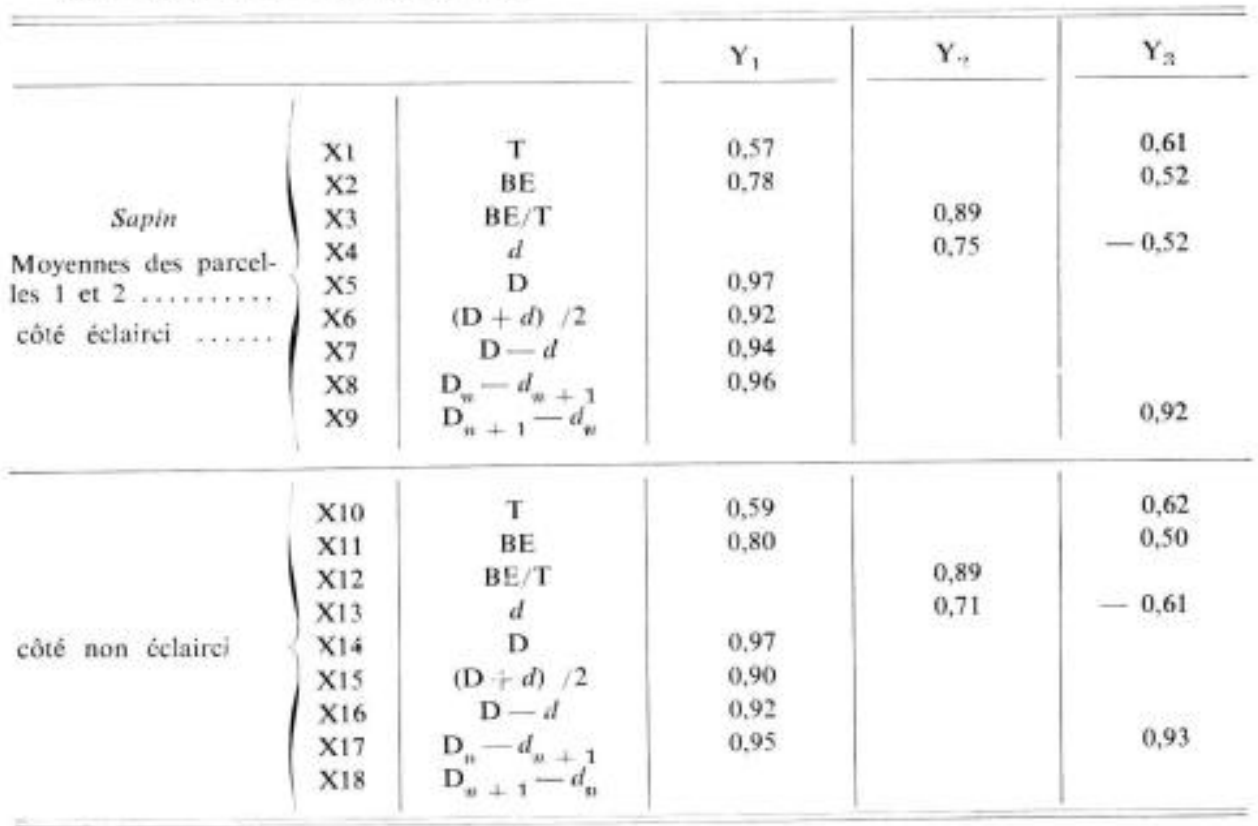

Ce qui s'observe sur le côté éclairci, s'observe également sur le côté non éclairci avec une similitude frappante.

La composante Y1 est très fortement liée avec les termes qui font intervenir la densité maximale $\mathrm{D} n$, la liaison la plus forte étant d'ailleurs obtenue avec le terme $\mathrm{D} n$ considéré seul. Elle est moins liée avec la largeur de bois d'été BE ou avec la largeur de cernes $T$.

$\mathrm{Y} 2$ est liée avec le rapport $\mathrm{BE} / \mathrm{T}$ et avec $d ; \mathrm{Y} 3$ est très fortement en corrélation avec l'écart de densité entre deux saisons de végétation $\mathrm{D} n+1-d n$ et d'une façon moindre avec T, BE et $d$.

On retrouve sur ce tableau ce que l'on avait tiré des calculs de corrélation entre variables initiales chez le sapin : la liaison positive de T, BE et D, les liaisons évidentes entre les combinaisons des $\mathrm{D} n$ avec $d n$, la liaison négative entre $\mathrm{D} n+1-d n$ et $d n$, qui n'apparaissent pas dans le cas de l'épicéa. 
Au lieu de conserver 9 variables initiales, il est probable qu'on aurait pu se contenter de $\mathrm{D} n, \mathrm{BE} / \mathrm{T}$ et $\mathrm{D} n+1-d n$ pour différencier les années. Si l'on regroupe les deux côtés, éclairci et non éclairci, ainsi que les deux parcelles, 88 \% de la variation entre les années sont expliqués par deux composantes principales.

\section{2. - Représentation graphique des années dans le système daxes des composantes}

\subsection{1. - Cas de deux composantes: le sapin.}

La représentation graphique (figure 4) des 26 années met en évidence des points isolés comme ceux qui correspondent à 1941, 1947, 1957, 1961, 1965, ou au contraire des groupements, tels 1942, 1943, 1946 ou 1949 et 1959, ou encore 1951 et 1955.

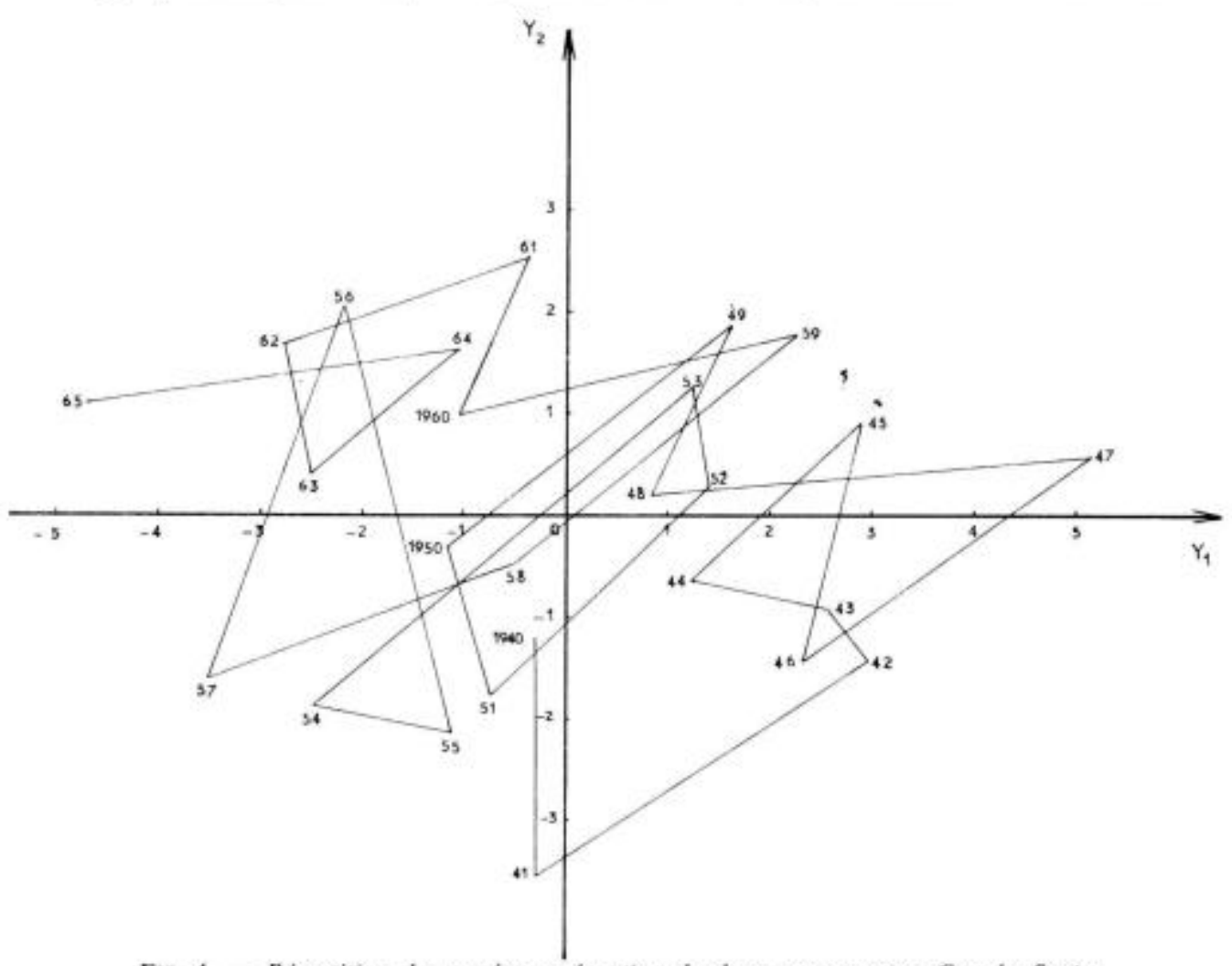

Fic, 4. - Répartition des années en fonction de deux composantes. Cas du Sapin Fic. 4. - Repartition of the years relatively to 2 components (Silver Fir)

Si l’on veut avoir une idée de la distance qui sépare les années, il est possible de se servir des valeurs des composantes principales qui sont les coordonnées des différents points représentatifs des années dans le système d'axes orthogonaux supportant ces composantes. Pour tenir compte de la valeur relative de chaque composante dans l'explication de la variabilité entre les années, il est nécessaire d'introduire les valeurs 
propres de ces composantes pour pondérer les termes carrés, dont la somme est égale au carré de la distance entre les deux années considérées. Si l'on se limite au cas simple où l'on n'a que deux composantes $\mathrm{Y} 1$ et $\mathrm{Y} 2$ de valeurs propres $\lambda 1$ et $\lambda 2$, la distance séparant l'année A de l'année B est donnée par l'égalité :

$$
\overrightarrow{\mathrm{AB}^{2}}=\frac{(b-a)^{2}}{\lambda .1}+\frac{\left(b^{\prime}-a^{\prime}\right)^{2}}{\lambda 2}
$$

Ce calcul est généralisable à un nombre quelconque de composantes, puisqu'elles sont orthogonales deux à deux.

Sur le graphique, on peut observer en outre les sauts qui font passer d'une année à la suivante. Certains s'effectuent parallèlement, ou presque, à l'une des composantes, comme dans le cas du passage de 1940 à 1941 ou de 1952 à 1953 ; d'autres le font parallèlement à la première bissectrice, comme de 1941 à 1942,1944 à 1945,1946 à 1947.1949 à 1950,1951 à 1952,1953 à 1954,1958 à 1959,1963 à 1964.

On pourrait se poser la question de savoir s'il n'apparaît pas une périodicité dans la disposition relative des points. Si l'on conserve tout d'abord l'exemple du sapin, où la représentation est simple du fait qu'il n'y a que deux composantes Y1 et Y2 dans le cas où tout est regroupé, côté non éclairci et côté éclairci et parcelles, on peut remarquer que, outre le parallélisme entre certains sauts d'une année à l'autre, il semble exister une période qui serait de dix à douze ans et qui sépare deux sauts parallèles et de même sens.

Ainsi, le passage de 1940 à 1941,1950 à 1951,1963 à 1964 s'effectue parallèlement à l'axe de Y2, vers les Y2 décroissants. Pour aller de 1941 à 1942, 1951 à 1952, 1964 à 1965 , il faut suivre une direction sensiblement parallèle à la première bissectrice, dans le sens des Y croissants. Le passage de 1942 à 1943 est approximativement parallèle et de même sens que celui qui fait aller de 1952 à 1953 ; on peut faire une remarque analogue en ce qui concerne le trajet de 1948 à 1949 et 1960 à 1961 ou 1958 à 1959. Il y a aussi des trajets qui sont parallèles et séparés par un espace de temps plus court et dont les rapports les uns avec les autres ne sont pas clairs. On peut cependant formuler une hypothèse : ces trajets parallèles pourraient être le reflet de variations des conditions climatiques que subissent les arbres et qui sont traduites et intégrées dans les composantes, Ces conditions se renouvelleraient périodiquement et il serait tentant d'associer à la période de $10-12$ ans la période des taches solaires qui est du même ordre de grandeur.

\subsection{2. - Cas de trois composantes : l'épicéa.}

L'épicéa fournit des résultats comparables mais, comme la différenciation des années a nécessité trois composantes principales Y1, Y2, Y3, il faut examiner les trajets d'une année à la suivante dans deux plans différents (figure 5).

Dans le plan des composantes Y1 et Y2, les trajets de 1941 à 1942, 1951 à 1952, 1960 à 1961 sont parallèles à $\mathrm{Y} 1$ et dirigés vers les $\mathrm{Y} 1$ décroissants ; on trouve un phénomène analogue pour le passage de 1943 à 1944 et de 1953 à 1954 , pour celui de 1946 à 1947 et de 1956 à 1957, ainsi que pour celui de 1948 à 1949 et de 1958 à 1959 . Or, dans le plan de $\mathrm{Y} 1$ et $\mathrm{Y} 3$, bien que le parallélisme entre les divers trajets ne soit plus aussi net, ce sont les mêmes années que l'on retrouve, séparées par des sauts de même sens. Chez l'épicéa aussi, on peut observer des trajets d'année à année parallèles, 

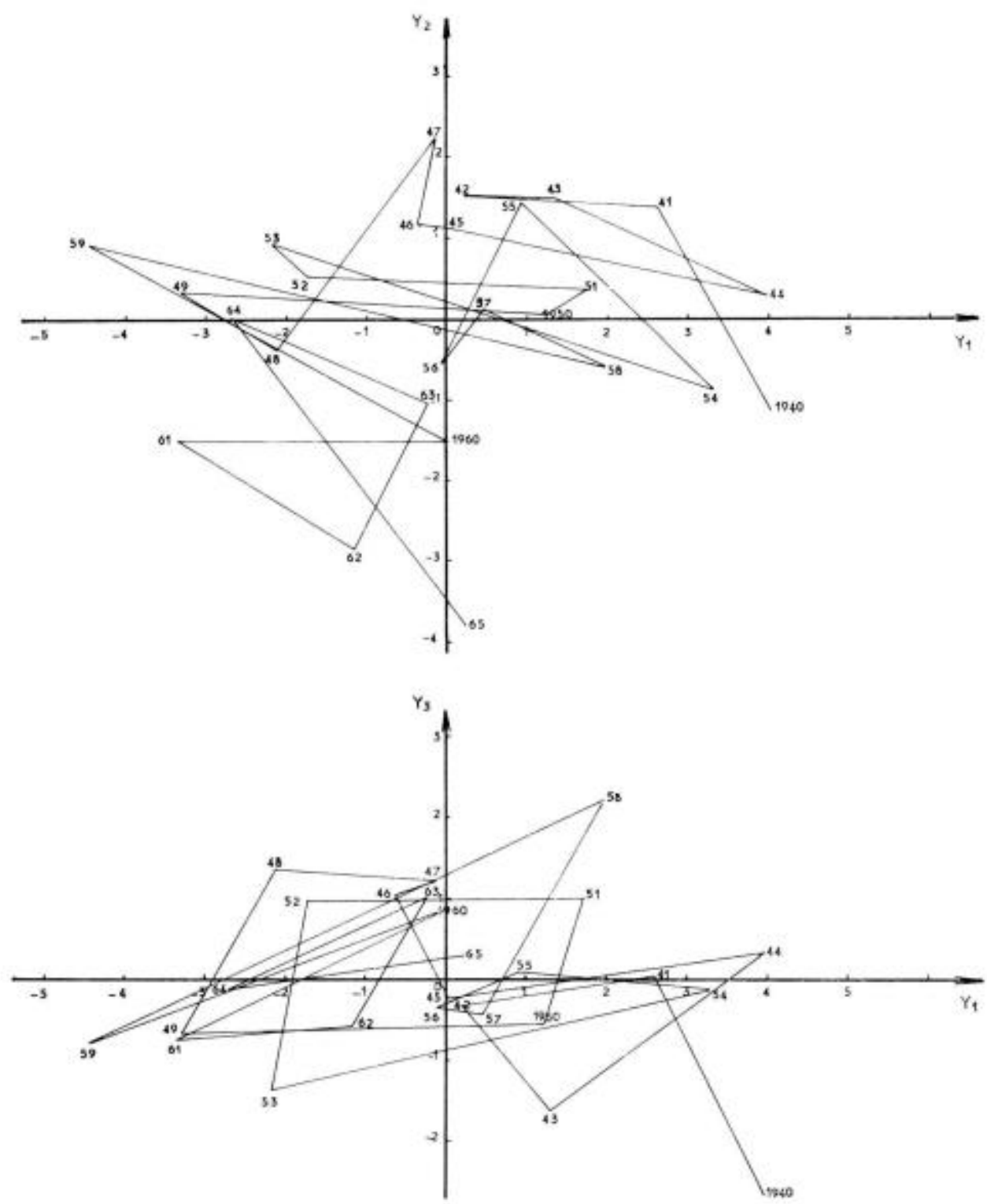

FIG. 5. - Répartition des années en fonction de trois composantes. Cas de lÉpicéa

Fic, 5. - Repartition of the years relatively to 3 components (Norway Spruce)

mais séparés par un petit nombre d'années, ainsi 1958 à 1959,1960 à 1961,1963 à 1964.

Comme couples d'années remarquables communs au sapin et à l'épicéa, on ne relève que 1941 à 1942 et 1951 à 1952 , ainsi que peut-être 1948 à 1949 et 1958 à 1959 . Il semble que l'analyse des composantes principales fasse ressortir chez l'épicéa une périodicité d'une dizaine d'années, semblable à celle du sapin et s'appliquant à des cou- 
ples d'années communs aux deux essences, ou non, de la même façon que les années caractéristiques trouvées au début de cette étude par la prise en considération des caractéristiques isolées ont pu être communes aux deux essences, ou au contraire différentes.

\subsection{3. - Années particulières.}

Intuitivement, on pourrait penser que les années périphériques des nuages de points représentés sur les graphiques des composantes principales sont remarquables à certains aspects, et auraient pu être découvertes dans l'analyse de la première partie où les caractéristiques étaient examinées une à une.

Chez le sapin, les années périphériques sont 1941, 1947, 1957, 1961 et 1965 dont trois sont les mêmes que celles qui ont été trouvées dans la première partie $(1947,1957$ èt 1965).

Dans le cas de l'épicéa, on peut noter 1940, 1944, 1953, 1954, 1959, 1961, 1962. dont cinq sont communes avec celles du tableau récapitulant les résultats de la première partie $(1944,1954,1959,1961$ et 1962).

\section{CONCLUSION}

L'analyse des composantes principales peut servir à la découverte d'années particulières, non seulement parce qu'elle permet d'observer des années qui se détachent des autres dans les représentations graphiques des nuages de points, mais aussi parce qu'elle apporte, à partir des variables initiales et par leur transformation, le maximum de variation entre toutes les années, si bien aue, à la limite, on peut penser que toutes les années sont caractéristiques, puisqu'individualisées dans l'espace des composantes.

On a vu que des années éloignées dans le temps pouvaient se grouper dans cet espace, que des années voisines étaient séparées par une grande distance, et aussi que le passage d'une année à la suivante semblait dans quelques cas se répéter identique à luimême à des intervalles de temps assez constants, ce qui peut suggérer que l'analyse des composantes principales, qui intègre plusieurs facteurs, fait ressortir d'une façon simple les réactions de l'arbre face aux éléments extérieurs ; ces réactions se traduiraient sur les graphiques par des directions privilégiées qui sont chacune l'image globale de l'évolution des caractères mesurés, provoquée par des conditions de milieu changeantes et périodiques. Chaque direction remarquable orientée dans un sens donné est l'image de la réaction de l'arbre soumis au passage de conditions extérieures données de l'année $n$ à un autre ensemble de conditions extérieures de l'année $n+1$. Le fait que ces directions orientées se retrouvent à des intervalles de temps égaux identiques à elles-mêmes, et souvent de même amplitude, peut être une vérification de la périodicité dans l'apparition et la succession des conditions de milieu. Dans cette étude, on a trouvé des intervalles de dix à douze ans qui, on l'a vu, font penser à la durée du cycle des taches solaires.

En outre, la méthode des composantes principales devrait pouvoir recevoir une application dans le domaine de la xylochronologie. A la largeur des cernes, qui est le seul facteur pris en considération en dendrochronologie, la xylochronologie ajoute des 
caractéristiques qui sont au moins aussi sensibles au milieu que l'accroissement annuel sur le diamètre, et dont le nombre n'est limité que par l'intérêt qu'il y a à n'utiliser que des variables indépendantes; cette méthode mathématique de séparation des observations à partir des variables initiales semble convenir à la résolution des problèmes de datation des bois, même quand le nombre d’années que l'on peut étudier n’est pas très élevé. Le faible nombre des observations est compensé par un nombre important de variables mesurées (largeur de cernes, plus les caractéristiques densitométriques).

On pourrait l'utiliser, soit pour synchroniser un échantillon inconnu avec un échantillon de référence, soit pour déterminer la place de cernes manquants d'un échantillon vis-à-vis d'un échantillon de référence complet. Les opérations seraient conduites de la façon suivante:

Supposons que l'on ait $\mathrm{N}$ arbres constituant un échantillon de référence synchrone de $\mathrm{A}$ années. Chacune de ces $\mathrm{A}$ années est caractérisée par $p$ mesures de variables initiales $\mathrm{X}_{1}, \mathrm{X}_{2}, \ldots, \mathrm{X}_{p}$ (par exemple : $\mathrm{X}_{1}=\mathrm{T}, \mathrm{X}_{2}=\mathrm{D}, \mathrm{X}_{3}=d$, etc...).

Supposons en outre que l'échantillon à synchroniser par rapport aux $\mathrm{A}$ années de référence n'ait que $n$ années consécutives comprises dans les $\mathrm{A}$ années $(n<\mathrm{A})$; il y a $\mathrm{A}-n+1$ possibilités de le placer par rapport à l'échantillon de référence, dont l'une est celle que l'on cherche (synchronisation exacte).

\section{Première méthode}

Le calcul des coefficients de corrélation sur les $n$ couples de variables prises 2 à 2 permet d'établir $\mathrm{A}-n+1$ matrices de ces coefficients (matrices carrées symétriques à $p$ lignes et $p$ colonnes, la diagonale étant constituée de 1). De chacune de ces matrices, on tire $p$ valeurs propres $\lambda_{1}, \lambda_{2}, \ldots, \lambda_{p}$. telles que $\lambda_{1}+\lambda_{2}+\ldots+\lambda_{p}=p$ et $p$ vecteurs propres $Y_{1}, Y_{2}, \ldots, Y_{p}$ orthogonaux 2 à 2 dont les composantes dans le système des variables initiales sont les $a_{i j}$ tels que :

$$
\begin{aligned}
& \mathrm{Y}_{1}=a_{11} \mathrm{X}_{1}+a_{12} \mathrm{X}_{2}+\ldots+a_{1 p} \mathrm{X}_{p} \\
& \mathrm{Y}_{p}=a_{p 1} \mathrm{X}_{1}+a_{p 2} \mathrm{X}_{2}+\ldots+a_{p p} \mathrm{X}_{p}
\end{aligned}
$$

Chaque fois que l'on appliquera aux variables initiales de l'échantillon à synchroniser les coefficients $a_{i j}$, on obtiendra les composantes de chacune des $n$ années de cet échantillon. Parmi les $\mathrm{A}-n+1$ cas possibles de comparaison de ces composantes avec celles de l'échantillon de référence, il en est un pour lequel les différences auront une valeur minimale. Ce dernier cas correspondra à la synchronisation cherchée, ou du moins l'espérons-nous.

S'il y a une année manquante chez l'échantillon à synchroniser, la coïncidence obesrvée sur 1 à $m$ années disparaîtra à partir de la $m+1$ ème année, ce qui pe:mettra immédiatement de fixer le rang à partir duquel une année fait défaut.

Dans cette méthode, les valeurs propres et les vecteurs propres sont supposés être les mêmes pour l'échantillon de référence et l'échantillon à synchroniser, les mêmes $a_{i j}$ servant aux calculs de leurs composantes. 


\section{Deuxième méthode}

Le calcul des coefficients de corrélation sur les $n$ couples de variables prises 2 à 2 de l'échantillon de référence de $\mathrm{N}$ individus permet d'établir $\mathrm{A}-n+1$ matrices carrées symétriques à $p$ lignes et $p$ colonnes. De chacune de ces matrices, on tire $p$ valeurs propres $\lambda_{1}, \lambda_{2}, \ldots, \lambda_{p}$ et $p$ vecteurs propres $\mathrm{Y}_{1}, \mathrm{Y}_{2}, \ldots, \mathrm{Y}_{p}$ dont les composantes dans le système des variables initiales sont les $a_{i j}$ tels que :

$$
\begin{aligned}
& \mathrm{Y}_{1}=a_{11} \mathrm{X}_{1}+\ldots+a_{p p} \mathrm{X}_{p} \\
& \mathrm{Y}_{p}=a_{p 1} \mathrm{X}_{1}+\ldots+a_{p p} \mathrm{X}_{p}
\end{aligned}
$$

Si l'on effectue le même calcul, mais cette fois en ajoutant l'échantillon à synchroniser aux $\mathrm{N}$ individus de l'échantillon de référence (donc sur $\mathrm{N}+1$ individus), on obtiendra des $a_{i j}^{\prime} \neq a_{i j}$ tels que :

$$
\left.\begin{array}{l}
\mathrm{Y}_{1}^{\prime}=a_{11}^{\prime} \mathrm{X}_{1}+\ldots+a_{1 p}^{\prime} \mathrm{X}_{p} \\
\mathrm{Y}_{p}^{\prime}=a_{p 1}^{\prime} \mathrm{X}_{1}+\ldots+a_{p p}^{\prime} \mathrm{X}_{p}
\end{array}\right\}
$$

et de nouvelles valeurs propres $\lambda_{1}^{\prime} \neq \lambda_{1} \lambda_{2} \neq \lambda_{2} \quad \lambda_{p}^{\prime} \neq \lambda_{p}$ ce qui se traduit par un déplacement des vecteurs propres par rapport à l'espace des composantes initiales (translation + rotation).

On peut penser que la synchronisation est réalisée lorsque dans les $\mathrm{A}-n+1$ comparaisons de l'état 1 à l'état 2 , on en trouvera une pour laquelle ce déplacement est minimal, c'est-à-dire lorsque les valeurs propres et les vecteurs propres de l'état 1 seront le moins différents des valeurs propres et des vecteurs propres de l'état 2 .

S'il y a une année manquante, la coïncidence observée entre $m$ années consécutives de l'état 1 et de l'état 2 cessera à partir des années numérotées $m+1$; c'est cette année $m+1$ qui justement manquera sur l'échantillon à synchroniser. Le même raisonnement peut être appliqué au cas de plusieurs années manquantes.

Nous espérons nous rendre compte de la validité de ces hypothèses à partir des données recueillies chez le Sapin et l'Epicéa, et nous pensons, au vu des premiers résultats que nous avons cités, mais qui sont très fragmentaires et qui devraient conduire à des recherches plus approfondies, que la méthode d'analyse des composantes principales doit faire progresser la xylochronologie, science de la datation des bois. Les nombreuses variables explicatives que la méthode densitométrique permet de mesurer facilement sont bien appropriées à ce type d'analyse qui met en évidence globalement des différences ou des analogies à partir d'un ensemble de résultats divers. 


\section{SUMMARY}

\section{UTILIZATION OF DENSITY COMPONENTS IN XYLOCHRONOLOGY}

The field of density components applications is wide. Here, they have been used to study the wood structure variations for 2 species: the Silver fir (Abies alba Mill.) and the Norway spruce (Picea abies (L.) Karst) growing together: two compartments of a mixed stand were concerned : they had been strongly damaged during the 1st world war. restored by plantation immediately after, and they had not been thinned up to 1957 and 1959.

100 firs and 100 spruces were studied by using as samples $5 \mathrm{~mm}$ increment cores: 2 samples were taken from each trees. one in the direction which had been most lightened by the thinning, the other in the opposite side (see fig. 1).

The cores were X-rayed by the routine method of our laboratory. The X-ray negatives were scanned by an automatic recording microdensitometer which gives the wood density variations within and between rings.

We have measured for each of 27 years (1940 to 1966) the ringwidth $T$, the percentage of ringwidth having a density higher than $550 \mathrm{~g} / \mathrm{dm}^{3}: \mathrm{BE} / \mathrm{T}$, the maximum annual density : $\mathrm{D}$, the minimum annual density : $d$, the mean value $\frac{\mathrm{D}+d}{2}$, the density contrast $\mathrm{D}-d$ within each ring, the difference $\mathrm{D} n-d n+1$ between the maximum density of the year $n$ and the minimum density of the year $n+1$, and the difference $D n+1-d n$ between the maximum density of the year $n+1$ and the minimum density of the year $n$.

The different values for each year were compared to those of the preceeding and of the following ones (see table 1). A year has been called s characteristic year * for a given feature when it varies in the same direction for at least $70 \%$ of the samples.

\section{I. - Xylochronology.}

\section{Results}

1. Ringwidth $\mathrm{T} n$ (see appendix 7 ).

For fir, 4 years, i.e. about $15 \%$ of the total number of years, are characteristic years. For spruce, this percentage is only $7,5 \%$. One year, 1955, is characteristic for both species.

\section{Maximum annual density $\mathrm{D} n$ (see appendix 2).}

We find for fir 3 new characteristic years (see table 5) different from the characteristic years observed for ringwidth. For spruce, 5 new characteristic years appear.

\section{Minimum annual density dn (see appendix 3).}

Separately, this feature don't give characteristic year. But together with maximum annual density it allows to have new characteristic year or to improve the coincidence percentages. Different combinations have been studied and have given the following results.

\section{Difference $\mathrm{D} n-$ dn (see appendix 4 ).}

Two new characteristic years appear for the fir (see table 7) giving a total number of 12 characteristic years. An additional characteristic year is found for spruce (see table 8).

\section{Mean value $\frac{\mathrm{D} n+d n}{2}$ (see appendix 5).}

This feature gives a new characteristic year as well for fir as for spruce (tables 9 and 10).

\section{Difference $\mathrm{D}_{N}-d_{w+1}$ (see appendix 7).}

The percentages which have been found are applied to the year $n+1$ (tables II and 12). Generally speaking, this year is characteristic when the year $n$ is characteristic for $\mathrm{D} n$ (see tables 15 and 16$)$.

\section{Difference $\mathrm{D}_{\nu+1}-$ dn (see appendix 8).}

This combination gives, by comparison with $\mathrm{D} n$ alone, 3 additional characteristic years (table 13) for fir. For spruce, it affords the highest percentage (table 14). 


\section{Proportion BE/T (see appendix 6).}

It gives no interesting result, perhaps because the reference level $550 \mathrm{~g} / \mathrm{dm}^{3}$ is too low in relation with the maximum annual densities $\mathrm{D} n$.

The results are summarized in the tables 15 and 16.

For fir, the ringwidth $\mathrm{T}$ alone gives $15 \%$ of characteristic years; together with the difference $\mathrm{D}_{n+1}-d_{n}$ this percentage is raised up to $37 \%$.

For spruce, we have only $7,5 \%$ of characieristic years with $\mathbf{T}$, but $26 \%$ when $\mathrm{Dn}$ is considered in addition with $\mathbf{T}$.

\section{II. - Correlations between the means values per tree of the different characteristics (see appendix 9 and 10 ).}

The ringwidth of spruce seems to increase when the percentage of dense wood decreases.

When the ringwidth of fir increases, the minimum annual density decreases and the maximum annual density increases, i.e. the within-ring heterogeneity of wood densicy becomes higher.

The table 17 shows the results of correlation analysis of the 26 mean annual values of the different characteristics between fir and spruce. dix 9).

The maximum annual density seems to be widely independant of the ringwidth (appen-

\section{III. - Main components analysis.}

In this study, each year is characterized by several data ; therefore it is possible to put them within a space of which the dimension is given by the number of these data, and, by the way of suitable transformations, to find new synthetic variables, obtained by linear combinations of the initial data and giving a better differenciation of the years.

The main components analysis shows some connections between these variables (see the tables 18 and 19 which gives the correlation coefficients between the initial variables and the main components). Furthermore it indicates the similarities or the oppositions between them; we see in particular the similarities of X5, X6, X7, X8, for both species, and also the opposition between $\mathrm{X} 1$ and $\mathrm{X} 4$ for spruce.

The diagrams 1 and 2 represent the years within the 2 dimensional space of the more discriminating main components, of which the number is 2 for fir and 3 for spruce. It appears some parallelisms in the way from one year to the following one, which could indicate a periodicity in the climatic conditions.

At last it is suggested that density components might be used to synchronize samples with a reference series and also to find missing rings, but this is to be verified in the practice.

\section{ZUSAMMENFASSUNG}

\section{XYLOCHRONOLOGISCHE STUDIEN DURCH BENÜTZUNG DER KOMPONENTEN DER HOLZDICHTE}

Die Benätzung der Komponenten der Holzdichte erlaubt es neue Kriterien in die Datierungsverfahren einzufüren. Vorliegende Studie möchte zeigen wie interessant es sein kann der Jahrringbreite densitometrische Eigenschaften hinzuzufügen, die man durch eine radiographische Analyse erhält.

Zwei Holzarten wurden in Betracht genommen: die Tanne (Abies pectinala D.C.) und die Fichte (Picea excelsa Link.); die Holzproben wurden mit einem Presslerschen Zuwachsbohrer (Durchmesser $5 \mathrm{~mm}$ ) eninommen und zwar in einem Waldgebiet wo beide Holzarten gemischt wachsen.

Die xylochronologische Studie zeigt, dass die Jahrringbreite was die Tanne betrifft $15 \%$ Charakteristischerjahre liefert. Betrachtet man darüber hinaus den Unterschied zwischen der maximalen Dichte des Jahres $n+1$ und der minimalen Dichte des Jahres $n$, dann erreicht der Prozentsatz der charakteristischen Jahre $37 \%$. Bei der Fichte ist das Ergebnis 7,5\% wenn man lediglich die Jahrringbreite in Betracht zieht und $26 \%$ wenn man dieser Angabe die maximale Jahresdichte hinzufïgt. 
Ein Jahr ist als charakteristisch angenommen worden, wenn sein Koinzidenzprozent $70 \%$ überschreitet. Die Jahrringe von 27 Jahren wurden gemessen. Was die Fichte betrifft vermochten die densitometrischen Daten allein Prozentsatze von über $80 \%$ zu liefern.

Da für jedes Jahr mehrere Kriterien verfügbar waren, wurden auch die Beziehungen zwischen den einzelnen bestimmt und die Hauptkomponenten analysiert. Man hofft, dass ein solches Rechnungsverfahren für die Datierung von Probestücken angewendet werden könnte sowie für die Bestimmung der Stelle wo Jahrringe vermisst werden und zwar durch Vergleich mit einer xylochronologischen Kontrollserie.

\section{REFERENCES BIBLIOGRAPHIQUES}

Polge H., 1966. Etablissement des courbes de variation de la densité du bois par exploration densitométrique de radiographies d'échantillons prélevés à la tarière sur des arbres vivants. Applications dans les domaines technologique et physiologique. Thèse Docteur ès Sciences Appliquées, Faculté de Sciences, Université de Nancy, 27 juin.

Polge H. Kel.lek R., 1968. Influence de lapprovisionnement en eau sur la structure interne des accroissement annuels. Expérience dirrigation sur Pins sylvestres. Annales des Sciences Forestières, 25 (3), 125-133.

Polge H., Keller R., 1969. La xylochronologie, perfectionnement logique de la dendrochronologie. Annales des Sciences Forestières, 26 (2), 225-256.

Tomassone R., 1965. Lanalyse des composantes principales. Note Scientifique, $n^{\circ}$ 1, Station de Biométric, C.N.R.F.

Tomassone R. Elèments d'analyse statistique à plusieurs variables. Notl Scientifique interne. Station de Biométrie, C.N.R.F. 
ANNEXE 1. - Courbes de lévolution des largeurs de cernes par parcelle, par essence et par côté APPENDIX 1. - Variations of ringwidth for each plor, each species and each side
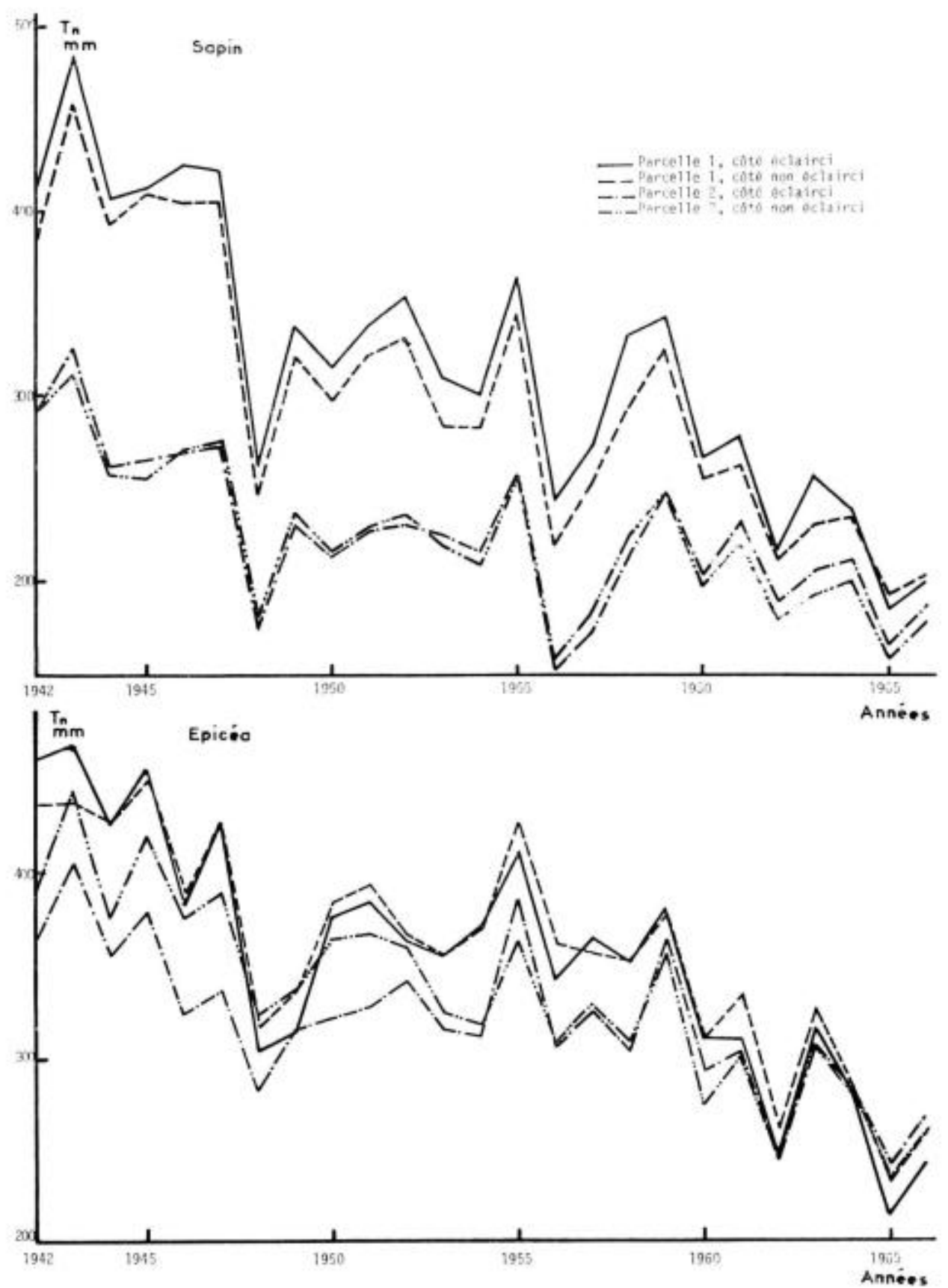
ANNEXE 2, - Courbes de févolution de la densité maximale $\mathrm{D}_{\text {A }}$ par parcelle, par essence et par côté Appenoix 2. - Variations of maximum anmad density $\mathrm{D}_{n}$ for each plot, each species and each side
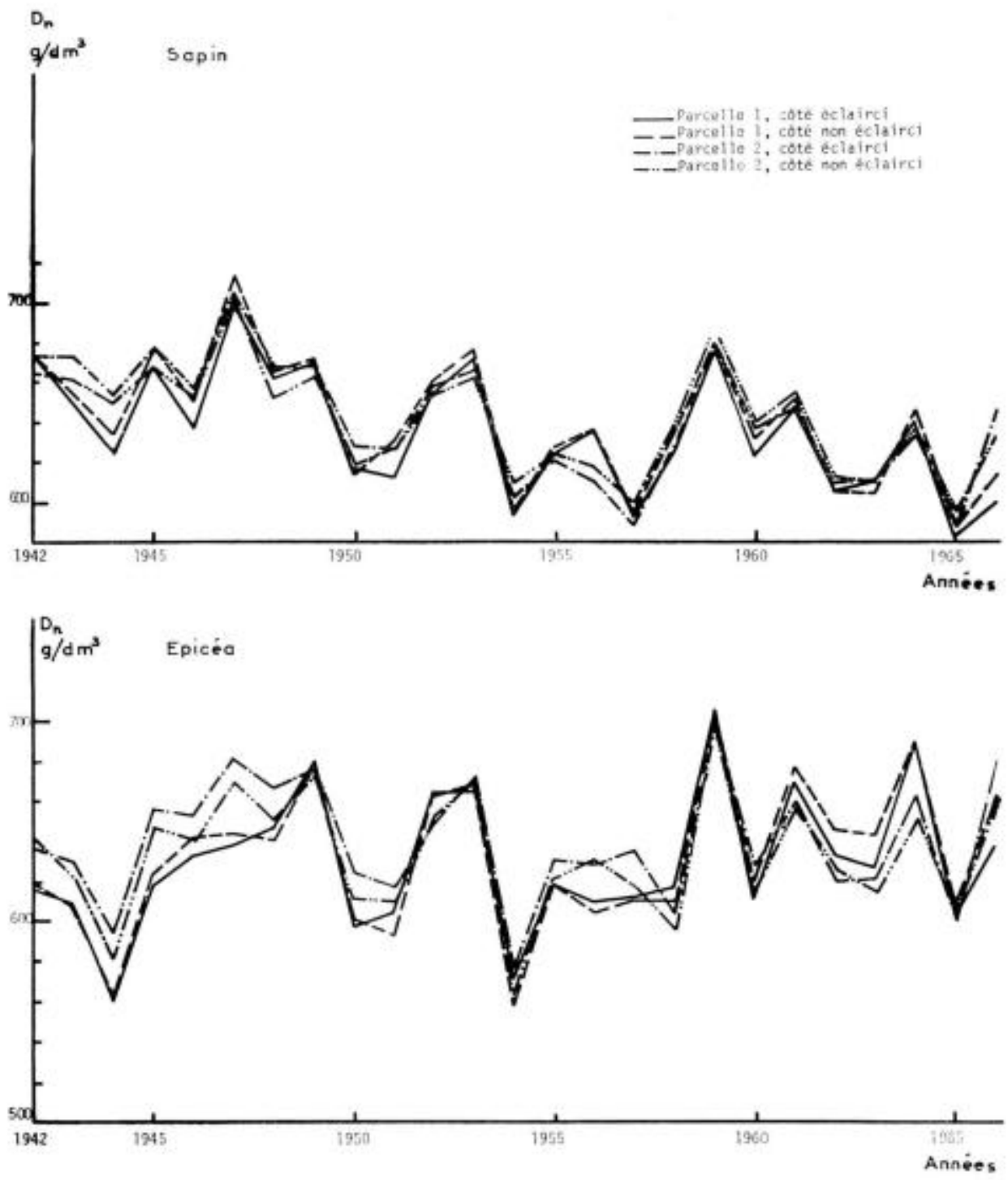
ANNEXE 3. - Courbes de Tévolution de la densité minimale $d_{n}$ par parcelle, par essence et par côté Appendix 3, - Variations of minimum annual density $d_{n}$ for each plot, each species and each side
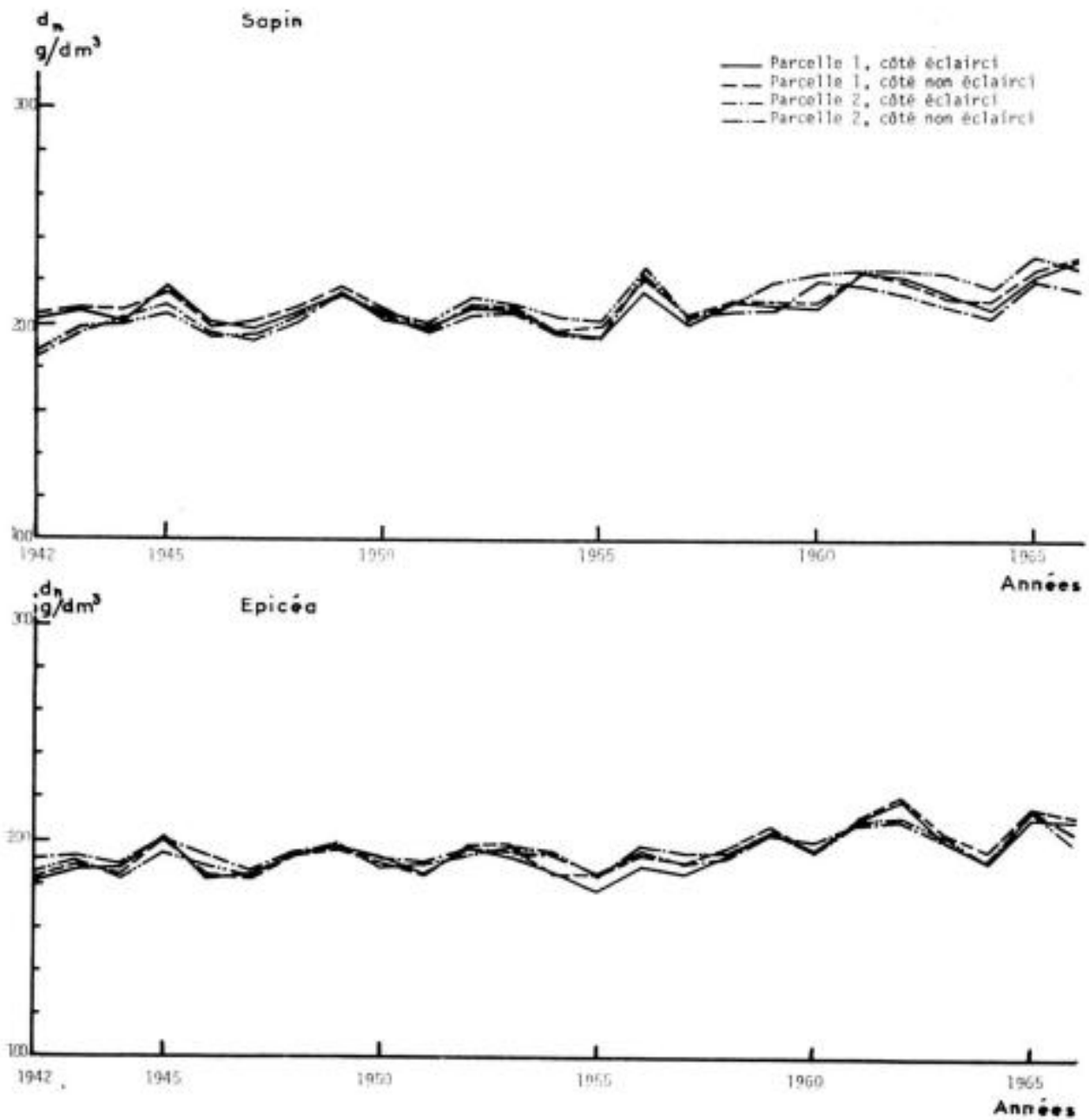
ANNEXE 4. - Courbes de l'évolution de $\mathrm{D}_{n}-d_{n}$ par parcelle, par essence et par côté Appendix 4. - Variations of $\mathrm{D}_{n}-d_{n}$ for each plot, each species and each side
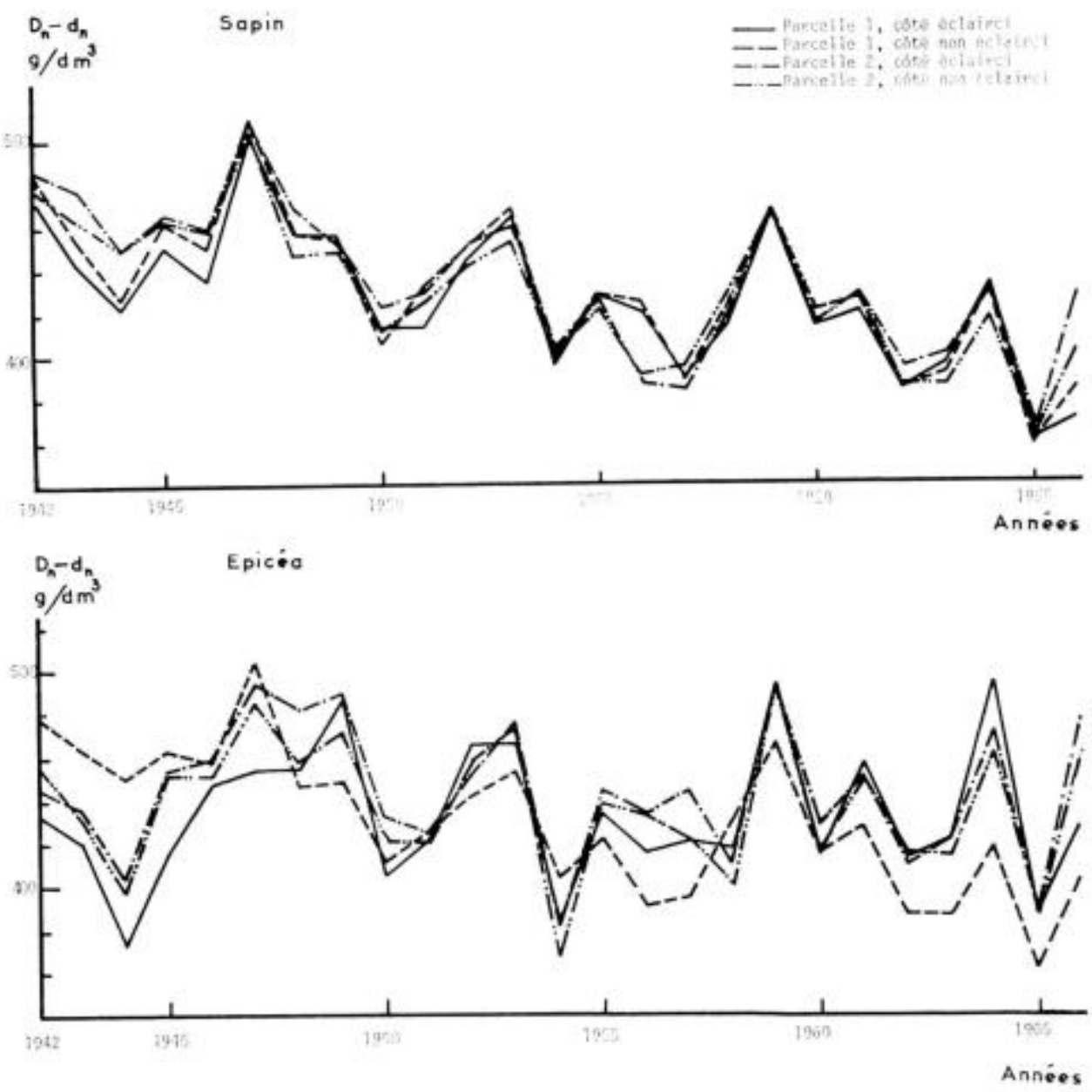
ANNEXE 5. - Courbes de tévolution de $\left(\mathrm{D}_{n}+d_{n}\right) / 2$ par parcelle, par essencé et par côté APPENDix 5. - Variations of $\left(\mathrm{D}_{w}+d_{w}\right) / 2$ for each plot, each species and each side
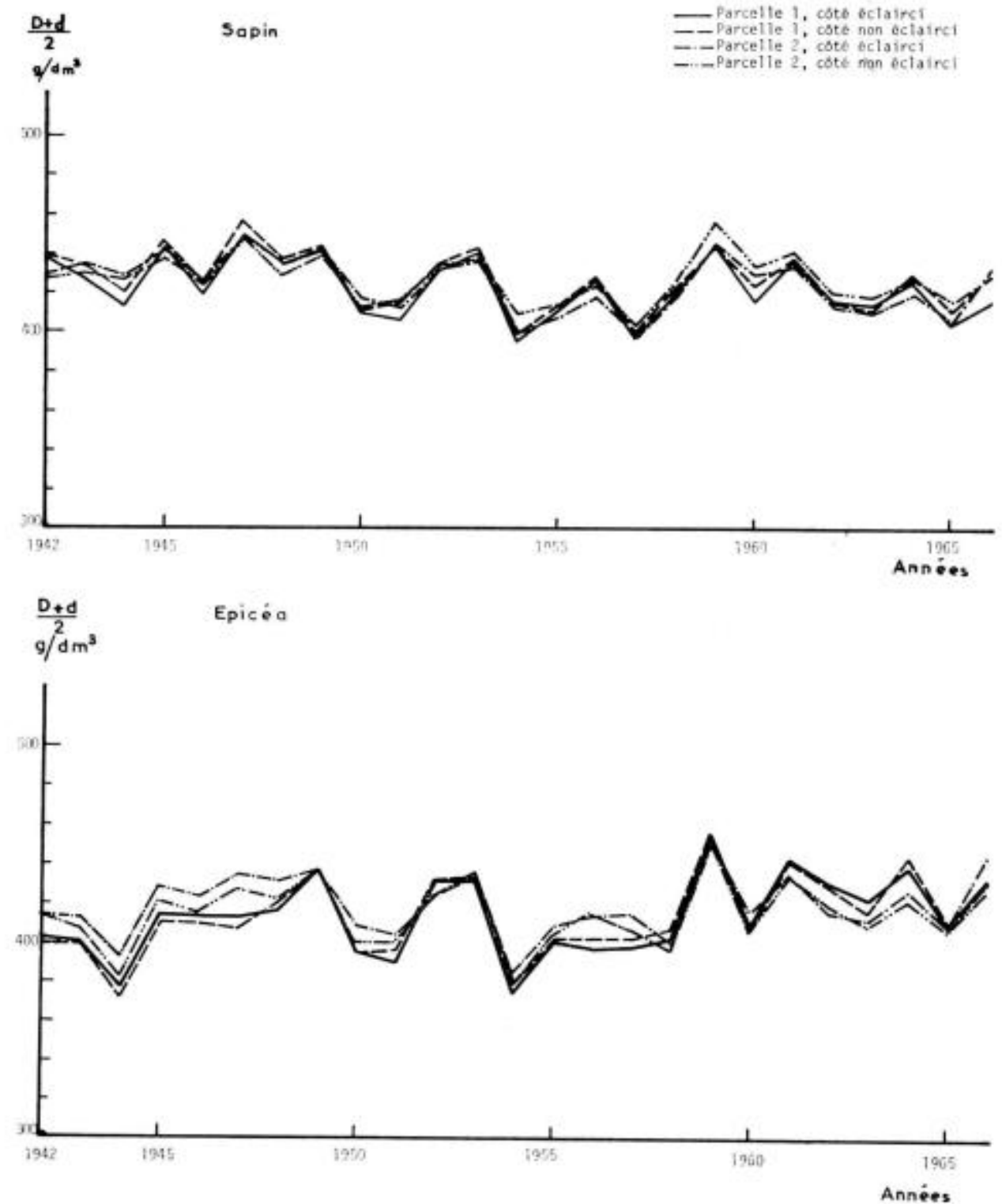
ANNExe 6. - Courbes de lévolution de BE/T par parcelle, par essence et par côté APPENDIX 6, - Variations of latewood percentage for each plot, each species and each side
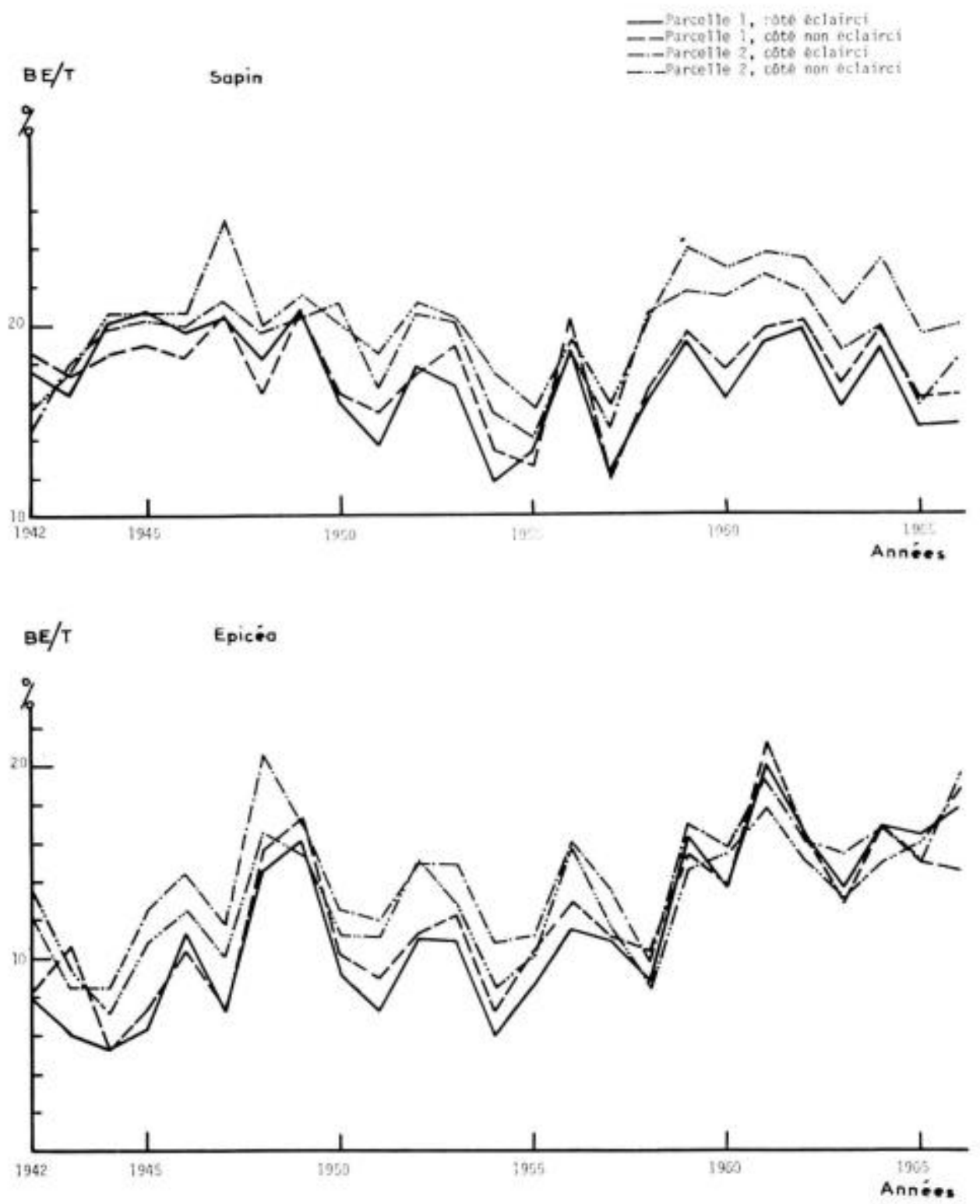
COMPOSANTES DE LA DENSITÉ ET XYLOCHRONOLOGIE

193

ANNEXE 7. - Courbes de lévolution de $\mathrm{D}_{n}-d_{n+1}$ par parcelle, par essence et par côté APPENDIX 7. - Variations of $\mathrm{D}_{n}-d_{n+1}$, for each plot, each species and each side
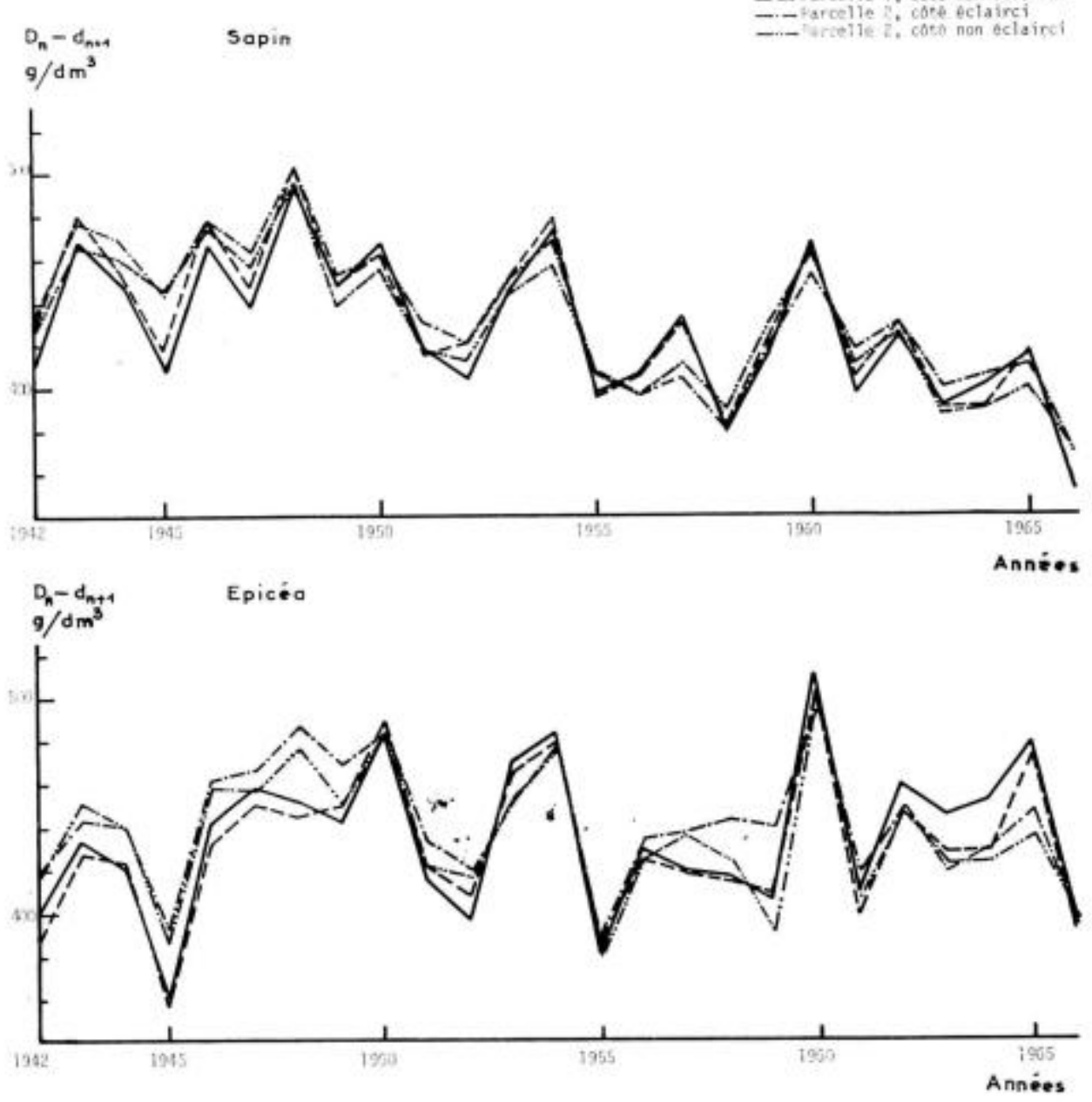
ANNEXE 8. - Courbes de lévolution de $\mathrm{D}_{n+1}-d_{n}$ par parcelle, par essence et par cóté APPENDIX 8. - Variations of $\mathrm{D}_{n+1}-d_{n}$ for each plot, each species and each side
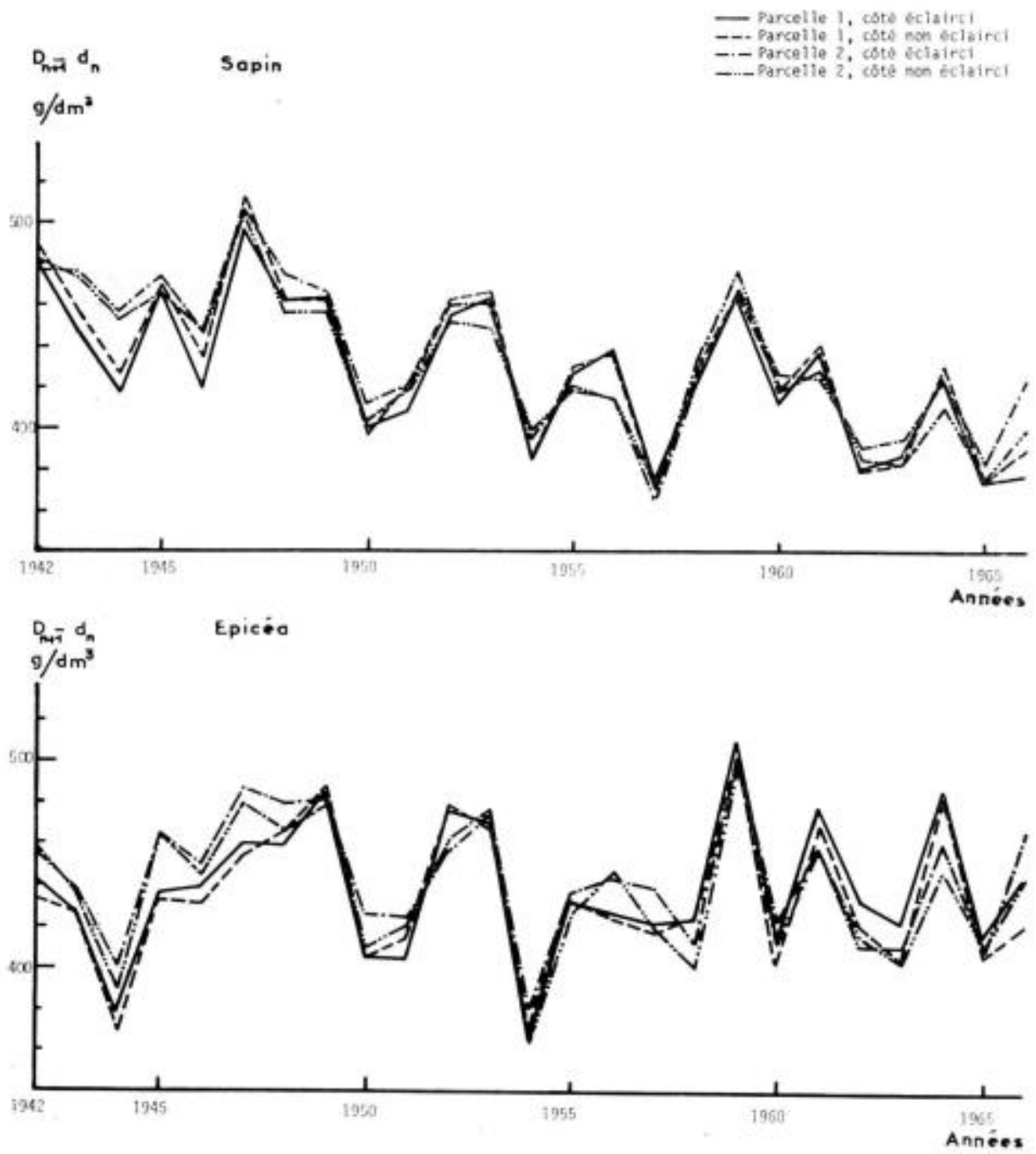
ANNEXE 9, - Coefficients de corrélation entre les diverses caractéristiques; seules les valeurs de $r>0,7$ ou de $r<-0,7$ ont été représentées

Appindix 9. - Correlation coefficients between the different characteristics (only the $r$ values higher than 0.7 or lower than $-0,7$ are represented)

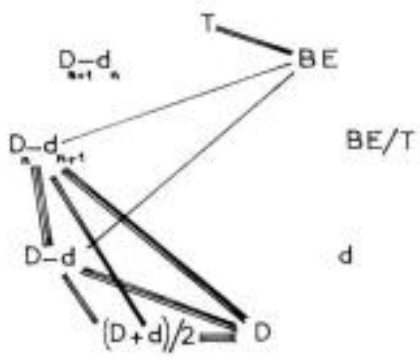

Parcelle 1

Cóté Ecrafiecs

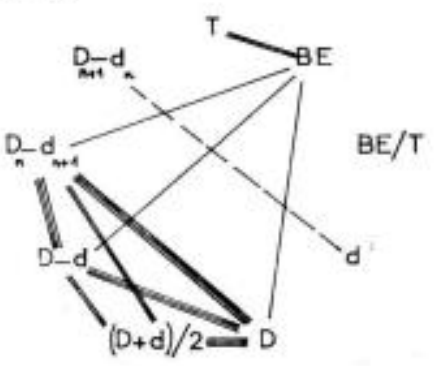

parcelle 1

Gótê non Eclairci

Sapin

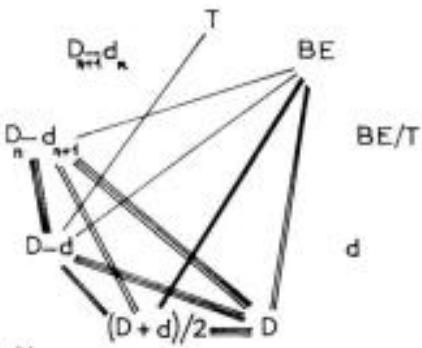

"arcelle I!

rote fclaircis

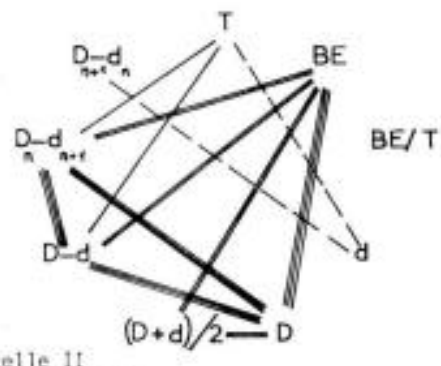

Parcelle II

Cóté nan Eclairci
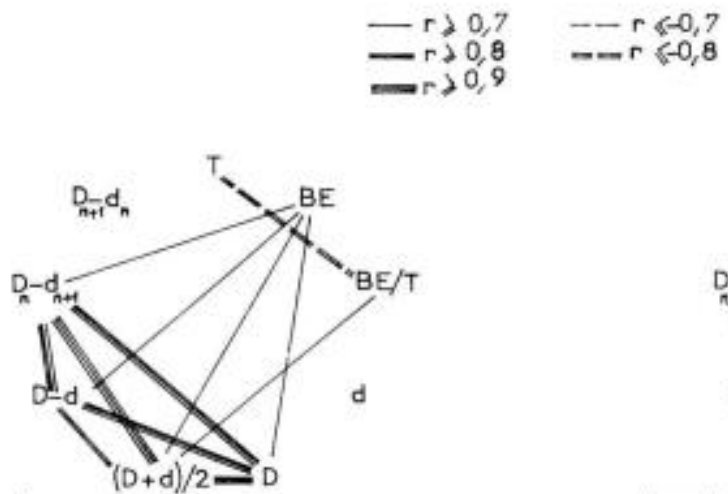

Parcelle 1

Coté Eclairci

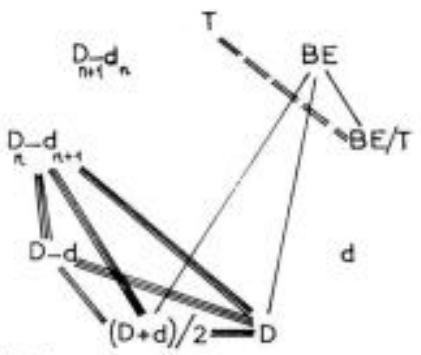

"yarcelle

cote nen Eclairci

Epicéo

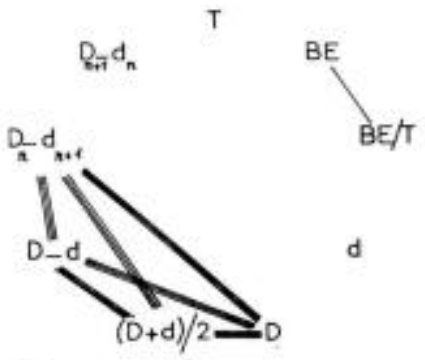


ANNEXE 10, - Coefficients de corrélation portant sur les moyennes des côtés et des parcelles APPENDIX 10. - Correlation coefficients between the mean values of both sides and of both plots

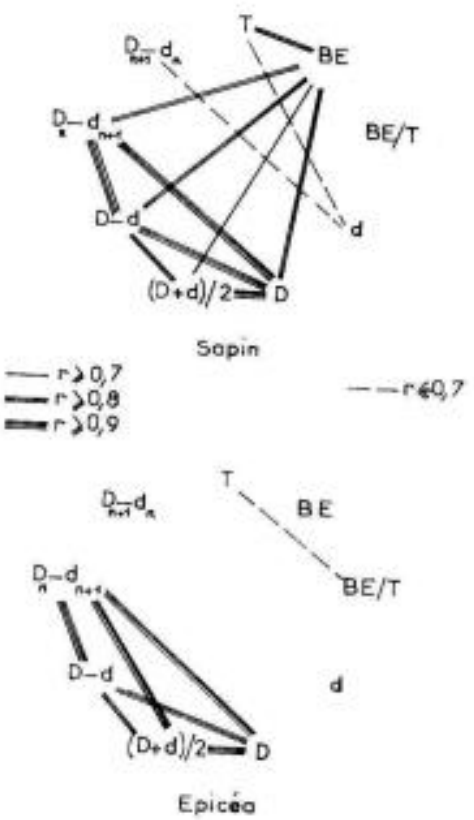

